\title{
ON CERTAIN INEQUALITIES AND CHARACTERISTIC VALUE PROBLEMS FOR ANALYTIC FUNCTIONS AND FOR FUNCTIONS OF TWO VARIABLES*
}

\author{
BY \\ KURT FRIEDRICHS
}

Part I. The case of analytic functions

\section{INTRODUCTION}

In this first part I investigate some properties of the manifold $\mathfrak{F}$ of all analytic functions $u+i v=w(z)$ defined in a bounded open connected domain $D$ of the $(z=x+i y)$-plane for which the integral

is finite. $\dagger$

$$
\iint_{D}|w|^{2} d x d y
$$

First I establish the following inequality. There exists a positive constant $\theta<1$ such that, for all functions $w(z)$ which satisfy the additional condition

$$
\iint w d x d y=0
$$

the inequality

$$
\left|\iint_{D} w^{2} d x d y\right| \leqq \theta \iint_{D}|w|^{2} d x d y
$$

is valid.

It will be seen that this inequality is equivalent to

$$
\iint_{D} u^{2} d x d y \leqq \Gamma \iint_{D} v^{2} d x d y
$$

under the additional condition

$$
\iint_{D} u d x d y=0
$$

the constant $\Gamma=(1+\theta) /(1-\theta)$ being greater than 1 .

* Presented to the Society, October 31, 1936; received by the editors December 6, 1935, and January 27, 1936.

† The same space was investigated in regard to different properties by St. Bergman, Mathematische Annalen, vol. 86 (1922), p. 238, and Berliner Sitzungsberichte, 1927, p. 178; and S. Bochner, Mathematische Zeitschrift, vol. 14(1922), p. 180). 
Secondly, I deal with the characteristic value problem for the quadratic form

$$
\iint_{D} w^{2} d x d y
$$

with respect to the unit-form

$$
\iint_{D}|w|^{2} d x d y
$$

I prove the existence of a sequence of characteristic values $\mu_{1}, \mu_{2}, \mu_{3}, \cdots, \mu_{n} \downarrow 0$, and corresponding characteristic functions $w_{1}(z), w_{2}(z), \cdots$ satisfying the conditions

$$
\iint_{D} \overline{w_{m}(z)} w_{n}(z) d x d y= \begin{cases}1, & n=m, \\ 0, & n \neq m,\end{cases}
$$

and thus being orthonormal, such that each function $w(z)$ in $\mathfrak{F}$ can be developed in a series

$$
w(z)=c_{1} w_{1}(z)+c_{2} w_{2}(z)+c_{3} w_{3}(z)+\cdots
$$

converging uniformly in every closed subdomain of $D$, while the expansions

$$
\begin{aligned}
& \iint_{D}|w|^{2} d x d y=\left|c_{1}\right|^{2}+\left|c_{2}\right|^{2}+\left|c_{3}\right|^{2}+\cdots \\
& \iint_{D} w^{2} d x d y=\mu_{1} c_{1}^{2}+\mu_{2} c_{2}^{2}+\mu_{3} c_{3}^{2}+\cdots
\end{aligned}
$$

hold.

The largest characteristic value $\mu_{1}$ is equal to 1 and the corresponding characteristic function $w_{1}(z)$ is constant. The inequality stated above expresses nothing but the fact that the second characteristic value $\mu_{2}$ is less than 1.

The validity of these theorems depends essentially on the nature of the boundary of the domain $D$. My assumption is that this boundary $B$ consists of a finite number of closed curves having a continuous tangent except at a finite number of corners. Then the inequality holds; but the expansion theorem is valid if and only if there are no corners (except internal cusps). In the case of corners the extreme points of the limit spectrum can be determined.

Let each closed curve of the boundary be represented by a continuous 
periodic function $z=z(s)$ of a parameter $s$. Except at a finite number of corners this function shall have a continuous derivative

$$
\frac{d z}{d s}=\dot{z}(s),
$$

which, at each corner, is continuous on both sides. We can assume

$$
|\dot{z}|=\left|\frac{d z}{d s}\right|=1
$$

so that $s$ is the arc length of the curve, and further that the normal $i \dot{z}$ is directed into the interior of $D$.

At a corner the argument of $\dot{z}$ has the jump $(1-\omega) \pi$, where $\omega \pi$ is the inner angle of the corner.

We assume $0<\omega \leqq 2$. Thus we exclude external cusps $(\omega=0)$; in this case it can happen that not even the inequality holds as we shall show at the end of this part.

\section{A BASIC LEMMA}

We set

$$
\mathbf{M}=\max _{\omega}\left|\frac{\sin \omega \pi}{\omega \pi}\right| .
$$

Since the case $\omega=0$ has been excluded we have $M<1$, and $M=0$ if there are no corners (except internal cusps).

LEMMA 1. Let $\epsilon$ be an arbitrary positive number. Then there exists a boundary strip $S$ in $D$, bounded by the exterior boundary $B$ and an interior boundary $B^{\prime}$ which consists of a finite number of rectifiable closed curves, such that for every function $w(z)=u(z)+i v(z)$ in $\mathfrak{F}$ the inequality

$$
\left|\iint_{S} w^{2} d x d y\right| \leqq(M+\epsilon) \iint_{S}|w|^{2} d x d y+\gamma \int_{B^{\prime}}|w|^{2}|d z|
$$

holds, $\gamma$ being a suitable positive constant depending on $\epsilon$. This inequality implies (2.2) $(1-\mathrm{M}-\epsilon) \iint_{S} u^{2} d x d y \leqq(1+\mathrm{M}+\epsilon) \iint_{S} v^{2} d x d y+\gamma \int_{B^{\prime}}|w|^{2}|d z|$.

Without loss of generality we may confine ourselves to the case of a domain $D$ bounded by only one closed curve $B$.

First we discuss the case of no corners. We may assume $\epsilon<1$. We choose a constant $\sigma$ in such a way that

$$
\left|\frac{z\left(s^{\prime}\right)-z(s)}{s^{\prime}-s}-\dot{z}(s)\right| \leqq \frac{\epsilon}{4} \text { as }\left|s^{\prime}-s\right| \leqq \sigma .
$$


We set

$$
\Delta z(s)=\frac{z(s+\sigma)-z(s)}{\sigma}, \quad \Delta \dot{z}(s)=\frac{\dot{z}(s+\sigma)-z(s)}{} .
$$

Then we have

$$
\begin{gathered}
|\Delta z(s)-\dot{z}(s)| \leqq \frac{\epsilon}{4}, \\
\frac{3}{4} \leqq|\Delta z(s)| \leqq \frac{5}{4} .
\end{gathered}
$$

We choose a number $\rho>0$ in such a way that

$$
\left|s^{\prime}-s\right| \leqq \sigma \text { as }\left|z\left(s^{\prime}\right)-z(s)\right| \leqq \rho
$$

and a number $T>0$ in such a way that

and

$$
T \leqq \frac{2}{5} \rho
$$

Consequently we have

$$
T\left|\frac{\Delta z\left(s^{\prime}\right)-\Delta z(s)}{s^{\prime}-s}\right| \leqq \frac{\epsilon}{40} \quad \text { as } \quad\left|s^{\prime}-s\right| \leqq \sigma .
$$

Now we introduce a new parameter $t, 0 \leqq t \leqq T$, and set

$$
z=z(s)+i t \Delta z(s) \text {. }
$$

The strip $0<t<T$ corresponds in a one-to-one way to a certain boundary strip $S$ in $D$, bounded by the boundary $B$ and an inner curve $B^{\prime}$ which correspond to $t=0$ and $t=T$ respectively. To show this we first prove the relation

(2.5) $\left|z^{\prime}-z-\left[\left(s^{\prime}-s\right)+i\left(t^{\prime}-t\right)\right] \dot{z}(s)\right| \leqq \frac{3}{8} \epsilon\left|\left(s^{\prime}-s\right)+i\left(t^{\prime}-t\right)\right|$,

as $\left|s^{\prime}-s\right| \leqq \sigma$.

In fact, in view of (2.3), (2.4), we have

$$
\begin{aligned}
\mid z^{\prime}-z- & {\left[\left(s^{\prime}-s\right)+i\left(t^{\prime}-t\right)\right] \dot{z}(s) \mid } \\
= & \mid\left[z\left(s^{\prime}\right)-z(s)-\left(s^{\prime}-s\right) \dot{z}(s)\right]+i t^{\prime}\left[\Delta z\left(s^{\prime}\right)-\Delta z(s)\right] \\
& +i\left(t^{\prime}-t\right)[\Delta z(s)-\dot{z}(s)] \mid
\end{aligned}
$$




$$
\begin{aligned}
& \leqq \frac{\epsilon}{4}\left|s^{\prime}-s\right|+\frac{\epsilon}{40}\left|s^{\prime}-s\right|+\frac{\epsilon}{4}\left|t^{\prime}-t\right| \\
& \leqq\left|\frac{\epsilon}{4}+\frac{\epsilon}{40}+i \frac{\epsilon}{4}\right|\left|\left(s^{\prime}-s\right)+i\left(t^{\prime}-t\right)\right| .
\end{aligned}
$$

Now let $z^{\prime}=z$. Then we have

$$
\begin{aligned}
\left|z\left(s^{\prime}\right)-z(s)\right| & =\left|t^{\prime} \Delta z\left(s^{\prime}\right)-t \Delta z(s)\right| \\
& \leqq t^{\prime}\left|\Delta z\left(s^{\prime}\right)\right|+t|\Delta z(s)| \\
& \leqq 2 T \frac{5}{4} \leqq \rho .
\end{aligned}
$$

Therefore $\left|s^{\prime}-s\right| \leqq \sigma$ and relation (2.5) leads to $\left|\left(s^{\prime}-s\right)+i\left(t^{\prime}-t\right)\right|\left(1-\frac{3}{8} \epsilon\right) \leqq 0$. Hence $s^{\prime}=s, t^{\prime}=t$. Thus the one-to-one correspondence of $0<t<T$ and $S$ is proved.

We calculate the Jacobian

$$
J=\frac{\partial(x, y)}{\partial(s, t)}=\Re \overline{\Delta z}(\dot{z}+i t \Delta \dot{z}) ;
$$

from $(2.3)^{\prime},(2.4)^{\prime}$ we find

$$
J \geqq 1-\frac{\epsilon}{4}-\frac{\epsilon}{32} \geqq \frac{1}{2} .
$$

In the strip $S$ the parameters $s$ and $t$ can be expressed in terms of $x, y$, hence in terms of $z=x+i y, \bar{z}=x-i y$; and a simple calculation yields the relation

$$
2 J \frac{\partial}{\partial z}=\overline{\Delta z} \frac{\partial}{\partial s}-i \overline{(\dot{z}+i t \Delta \dot{z})} \frac{\partial}{\partial t} .
$$

We now introduce the function

$$
j(z, \bar{z})=z(s)-i t \Delta z(s)
$$

and find

$$
J \frac{\partial j}{\partial z}=i \Im(\dot{z} \overline{\Delta z})+t \Im(\Delta \dot{z} \overline{\Delta z}) .
$$

From $(2.3)^{\prime},(2.4)^{\prime},(2.6)$ we get

$$
\left|\frac{\partial j}{\partial z}\right| \leqq \epsilon .
$$


By a simple calculation, we obtain the identity

$$
\begin{aligned}
\iint_{\tau<i<T}\left(1-\frac{\overline{\partial j}}{\partial z}\right) w^{2} d x d y= & \iint_{\tau<i<T} \frac{\partial}{\partial \bar{z}}\left[(\overline{z-j}) w^{2}\right] d x d y \\
=\frac{i}{2} \int_{B^{\prime}}(\overline{z-j}) w^{2} d z & -\frac{i}{2} \int_{t=\tau}(\overline{z-j}) w^{2} d z
\end{aligned}
$$

for $0<\tau<T$. Since $\int_{0}^{T} \int_{t=\tau}|w|^{2} d s d \tau \leqq 2 \iint_{S}|w|^{2} d x d y$ is finite, there exists a sequence $\tau \rightarrow 0$ for which $\int_{t=\tau}|w|^{2} d s$ is bounded. If we let $\tau$ tend to zero in this way, we have

$$
\begin{aligned}
\left|\frac{i}{2} \int_{t=\tau}(\overline{z-j}) w^{2} d z\right| & =\left|\int_{t=\tau} \overline{t z} w^{2}(\dot{z}+i t \Delta \dot{z}) d s\right| \\
& \leqq \tau\left(1+\frac{\epsilon}{2}\right) \int_{t=\tau}|w .|^{2} d s \rightarrow 0
\end{aligned}
$$

and therefore the identity

$$
\iint_{S}\left(1-\frac{\overline{\partial j}}{\partial z}\right) w^{2} d x d y=T \int_{B^{\prime}} w^{2} \overline{\Delta z} d z
$$

It yields immediately the inequality

$$
\left|\iint_{S} w^{2} d x d y\right| \leqq \frac{5}{4} T \int_{B^{\prime}}|w|^{2}|d z|+\epsilon \iint_{S}|w|^{2} d x d y .
$$

\section{CASE OF CORNERS}

We now pass on to the case of boundary $B$ having corners $z_{y}$ with the angles $\omega_{\nu} \pi(\nu=1, \cdots, n)$. We map the domain $D$ conformally on a domain $D^{*}$ of the $z^{*}$-plane such that the boundary $B^{*}$ of $D^{*}$ has a continuous tangent. There exists such a mapping, regular in $D+B$ except at the corners, which behaves at the corners as follows: there is an analytic function

$$
\lambda=\lambda(z) \text { with } \lambda\left(z_{\nu}\right)=0, \quad \lambda^{\prime}\left(z_{\nu}\right) \neq 0,
$$

regular in the neighborhood of $z=z_{\nu}$, and an analytic function

$$
\lambda_{*}=\lambda_{*}\left(z^{*}\right) \quad \text { with } \lambda_{*}\left(z_{\nu}^{*}\right)=0, \quad \lambda_{*}^{\prime}\left(z_{\nu}^{*}\right) \neq 0
$$

(where $z_{\nu}^{*}$ corresponds to the corner $z_{\nu}$ ), regular in the neighborhood of $z^{*}=z_{\nu}^{*}$, such that 


$$
\lambda=\lambda_{*}^{\omega_{\nu}} \text {. }
$$

We shall make use of the fact that there is a constant $\chi>0$ such that

$$
\left|\left(z^{*}-z_{\nu}^{*}\right) \frac{d}{d z^{*}} \log \frac{d z}{d z^{*}}+\left(1-\omega_{\nu}\right)\right| \leqq \chi\left|z^{*}-z_{\nu}^{*}\right|+\chi\left|z^{*}-z_{\nu}^{*}\right| \omega_{\nu}
$$

in a certain neighborhood $U_{\nu}^{*}$ of $z_{\nu}^{*}$. We see this by a simple calculation:

Since

$$
\begin{aligned}
\frac{d z^{*}}{d z} & =\frac{\lambda_{*}^{1-\omega_{\nu}}}{\omega_{\nu}} \frac{\lambda^{\prime}}{\lambda_{*}^{\prime}}, \\
\lambda_{*} \frac{d}{d \lambda_{*}} \log \frac{d z^{*}}{d z} & =\left(1-\omega_{\nu}\right)-\lambda_{*} \frac{\lambda_{*}^{\prime \prime}}{\left(\lambda_{*}^{\prime}\right)^{2}}+\omega_{\nu} \lambda \frac{\lambda^{\prime \prime}}{\left(\lambda^{\prime}\right)^{2}} .
\end{aligned}
$$

$$
\frac{z^{*}-z_{\nu}^{*}}{\lambda_{*}}: \frac{d z^{*}}{d \lambda_{*}}=1+\left(z^{*}-z_{\nu}^{*}\right) \vartheta_{*}\left(z^{*}\right)
$$

where $\vartheta_{*}\left(z^{*}\right)$ is bounded at $z^{*}=z_{p}^{*}$, we get

$$
\begin{aligned}
\left(z^{*}-z_{\nu}^{*}\right) \frac{d}{d z^{*}} \log \frac{d z^{*}}{d z}-\left(1-\omega_{\nu}\right)=(1 & \left.-\omega_{\nu}\right)\left(z^{*}-z_{\nu}^{*}\right) \vartheta_{*}\left(z^{*}\right)-\left(z^{*}-z_{\nu}^{*}\right) \frac{\lambda_{*}^{\prime \prime}}{\lambda_{*}^{\prime}} \\
& +\omega_{\nu}\left(z^{*}-z_{\nu}^{*}\right)^{\omega_{\nu}}\left(\frac{z^{*}-z_{\nu}^{*}}{\lambda_{*}}\right)^{1-\omega_{\nu}} \frac{\lambda_{*}^{\prime}}{\lambda^{\prime}} \frac{\lambda^{\prime \prime}}{\lambda^{\prime}} .
\end{aligned}
$$

Since $\lambda_{*} /\left(z^{*}-z_{\nu}^{*}\right) \rightarrow \lambda_{*}^{\prime}(0) \neq 0$ as $z^{*} \rightarrow z_{\nu}^{*}$ and $\lambda^{\prime}(0) \neq 0$, there is a number $\chi>0$ and a neighborhood $U_{v}^{*}$ of $z_{v}^{*}$, where

$$
\left|1-\omega_{\nu}\right|\left|\vartheta_{*}\right|+\left|\frac{\lambda_{*}^{\prime \prime}}{\lambda_{*}^{\prime}}\right| \leqq \chi, \quad \omega_{\nu}\left|\frac{z^{*}-z_{\nu}^{*}}{\lambda^{*}}\right|^{1-\omega_{\nu}}\left|\frac{\lambda_{*}^{\prime}}{\lambda^{\prime}}\right|\left|\frac{\lambda^{\prime \prime}}{\lambda^{\prime}}\right| \leqq \chi
$$

and, thus, (3.1) holds in $U_{v}^{*}$. We introduce the function

$$
\eta=\frac{d z}{d z^{*}}: \frac{\overline{d z}}{d z^{*}} \text { with }|\eta|=1 \text {. }
$$

† We can construct such a mapping, e.g., in the following way. We choose a number $a_{1}$ in the exterior of $D+B$ such that the function

$$
z^{(1)}=\left(\frac{z-z_{1}}{z-a_{1}}\right)^{1 / \omega_{1}}
$$

maps the domain $D+B$ in a one-to-one way on a domain $D^{1}+B^{1}$ of the $z^{(1)}$-plane; $D^{1}+B^{1}$ has no corner at the point $z^{(1)}=z_{1}{ }^{(1)}$. In the same way we choose $a_{2}^{(1)}$ in the exterior of $D^{1}+B^{1}$ and form

and so on. Then we set

$$
z^{(2)}=\left(\frac{z^{(1)}-z_{2}^{(1)}}{z^{(1)}-a_{2}^{(1)}}\right)^{1 / \omega_{2}},
$$

and take $\lambda_{*}=z^{(n)}$ for the corner $z=z_{\nu}$.

$$
z^{*}=z^{(n)}
$$


We observe that relation (3.1) is equivalent to

$$
\left|\left(z^{*}-z_{\nu}^{*}\right) \frac{\partial \eta}{\partial z^{*}}+\left(1-\omega_{\nu}\right) \eta\right| \leqq \chi\left|z^{*}-z_{\nu}^{*}\right|+\chi\left|z^{*}-z_{\nu}^{*}\right|^{\omega_{\nu}},
$$

where $\eta$ is considered as a function of $z^{*}$ and $\overline{z^{*}}$. On setting

we have

$$
w^{*}\left(z^{*}\right)=w(z) \frac{d z}{d z^{*}}
$$

$$
\begin{aligned}
\iint_{D}|w|^{2} d x d y & =\iint_{D^{*}}\left|w^{*}\right|^{2} d x^{*} d y^{*} \\
\iint_{D} w^{2} d x d y & =\iint_{D^{*}}\left(w^{*}\right)^{2} \bar{\eta} d x^{*} d y^{*}
\end{aligned}
$$

\section{Continuation}

In what follows we omit the sign * and write $z, z_{\nu}, D, B, S, U_{v}, w$ instead of $z^{*}, z_{\nu}^{*}, D^{*}, B^{*}, S^{*}, U_{\nu}^{*}, w^{*}$.

Let the boundary $B$ be represented by $z=z(s)$, and let $s_{\nu}$ be that value of $s$ for which $z\left(s_{v}\right)=z_{v}$; we set $\dot{z}\left(s_{v}\right)=\dot{z}_{\nu}$. We introduce the parameters $s$ and $t$ in the boundary strip as before $(\$ 2)$ and define the function $j(z, \bar{z})$.

$$
z=z(s)+i t \Delta z(s) ; \quad j=z(s)-i t \Delta z(s) .
$$

Let $S_{v}$ and $B_{v}$ be the domain of all points of $S$ and $B$ respectively for which $\left|s-s_{\nu}\right| \leqq \sigma$, except $z=z_{\nu}$. We assume $\epsilon<\frac{1}{3}$ and at the same time so small that $B,+S_{v}$ is contained within the neighborhood $U_{v}$ of $z=z_{v}$. From (3.2) we get

$$
\left|\left(z-z_{\nu}\right) \frac{\partial \eta}{\partial z}+\left(1-\omega_{\nu}\right) \eta\right| \leqq \chi\left|z-z_{\nu}\right|+\chi\left|z-z_{\nu}\right|^{\omega_{\nu}} \text { in } S_{\nu}+B_{\nu} .
$$

We take note of the relations

$$
\begin{aligned}
& \left|\left(z-z_{\nu}\right)-\left(s-s_{\nu}+i t\right) \dot{z}_{\nu}\right| \leqq \frac{3}{8} \epsilon\left|s-s_{\nu}+i t\right| \\
& \left|\left(j-z_{\nu}\right)-\left(s-s_{\nu}-i t\right) \dot{z}_{\nu}\right| \leqq \frac{3}{8} \epsilon\left|s-s_{\nu}-i t\right|
\end{aligned}
$$

which holds for $\left|s-s_{\nu}\right| \leqq \sigma$ and, therefore, in $S_{\nu}+B_{v}$. They can be derived in the same way as relation (2.5).

We define the functions

$$
\mathrm{K}_{\nu}(z, \bar{z})=-\frac{z-z_{\nu}}{\dot{z}_{\nu}}: \frac{j-z_{\nu}}{\dot{z}_{\nu}}
$$


in $S,+B$; we have

$$
\mathrm{H}_{\nu}(z, \bar{z})=-\frac{z-z_{v}}{\dot{z}_{\nu}}: \frac{\overline{z-z_{v}}}{\dot{z}_{\nu}}
$$

(i) $\left|H_{\nu}\right|=1$,

(ii) $1-\epsilon \leqq\left|K_{\nu}\right| \leqq 1+\epsilon$,

(iii) $\left|H_{\nu}-K_{\nu}\right| \leqq \epsilon$.

In view of (4.2) relation (iii) follows from

$$
\left|\mathrm{H}_{\nu}-\mathrm{K}_{\nu}\right|=\frac{\left|\frac{\overline{z-z_{\nu}}}{\dot{z}_{\nu}}-\frac{j-z_{\nu}}{\dot{z}_{\nu}}\right|}{\left|\frac{j-z}{\dot{z}_{\nu}}\right|} \leqq \frac{\frac{3}{4} \epsilon}{1-\frac{3}{8} \epsilon} \leqq \epsilon .
$$

The relations (iii) and (i) lead to (ii).

The function $\mathrm{K}_{\nu}$ has the value 1 on the lines $s=s_{\nu}, 0<t<T$, and the value -1 on the boundary $t=0, s \neq s_{v}$. Therefore $\log \mathrm{K}$, is defined; we have

$$
\begin{array}{llll}
\log \mathrm{K}_{\nu}=0 & \text { on } & s=s_{\nu}, & 0<t<T ; \\
\log \mathrm{K}_{\nu}=\mp i \pi & \text { on } & t=0, & s \lesseqgtr s_{\nu} .
\end{array}
$$

In view of (4.2) we get

$$
\left|\log K_{\nu}\right| \leqq \frac{\frac{3}{4} \epsilon}{1-\frac{3}{8} \epsilon}+\left|\log \frac{t+i\left(s-s_{\nu}\right)}{t-i\left(s-s_{\nu}\right)}\right| \leqq \epsilon+\pi .
$$

We can define $\log \mathrm{H}_{\nu}$ in such a way that $\left|\log \mathrm{H}_{\nu}\right| \leqq 3 \epsilon / 2$ on the line $s=s_{\nu}$ and we set

$$
\mathrm{K}_{\nu}^{\omega_{\nu}}=\exp \omega_{\nu} \log \mathrm{K}_{\nu}, \quad \mathrm{H}_{\nu}^{\omega_{\nu}}=\exp \log \mathrm{H}_{\nu} .
$$

From (iii) we obtain

$$
\text { (iv) }\left|\mathrm{K}_{\nu}{ }^{\omega_{\nu}}-\mathrm{H}_{\nu}{ }^{\omega_{\nu}}\right| \leqq 3 \epsilon \text {. }
$$

We consider the function

$$
\mathrm{P}_{\nu}(z, \bar{z})=\frac{1}{\omega_{\nu}} \mathrm{K}_{\nu} \omega_{\nu}-\frac{\cos \omega_{\nu} \pi}{\omega_{\nu}}-\frac{\sin \omega_{\nu} \pi}{\omega_{\nu} \pi} \log K_{\nu},
$$

defined in $S_{\nu}+B_{\nu}$; it vanishes on the boundary $t=0, s \neq s_{\nu}$, and it is bounded. Therefore we have

$$
\begin{aligned}
\left(z-z_{\nu}\right) \mathrm{P}_{\nu}(z, \bar{z}) & \rightarrow 0 \\
\left(z-z_{\nu}\right){ }^{\omega \nu} \mathrm{P}_{\nu}(z, \bar{z}) & \rightarrow 0
\end{aligned}
$$


uniformly in $s$. We calculate the derivative of $\mathrm{P}_{\boldsymbol{v}}$ with respect to $z$ and find

$$
\left(z-z_{\nu}\right) \frac{\partial \mathrm{P}_{\nu}}{\partial z}=\left[\mathrm{K}_{\nu}^{\omega_{\nu}}-\frac{\sin \omega_{\nu} \pi}{\omega_{\nu} \pi}\right]\left(1+\mathrm{K}_{\nu} \frac{\partial j}{\partial z}\right) .
$$

According to (ii), (iv) and

$$
\left|\frac{\partial j}{\partial z}\right| \leqq \epsilon
$$

we get

$$
\left|\left(z-z_{\nu}\right) \frac{\partial \mathrm{P}_{\nu}}{\partial z}-\mathrm{H}_{\nu}^{\omega_{\nu}}\right| \leqq\left|\frac{\sin \omega_{\nu} \pi}{\omega_{\nu} \pi}\right|+6 \epsilon .
$$

We choose non-negative functions $\rho_{\nu}(s)$ which have continuous derivatives, which vanish outside of $B_{\nu}$, which are equal to 1 for $\left|s-s_{\nu}\right| \leqq \sigma / 2$ and for which

$$
\sum_{\nu} \rho_{v}(s) \leqq 1
$$

Here the summation $\sum_{\nu}$ is extended over all points $z=z_{p}$. We consider the function

Since

$$
\Omega(z, \bar{z})=\sum_{\nu} \rho_{\nu}(s) \mathrm{H}_{\nu}^{-\omega_{\nu}}\left(z-z_{\nu}\right) \eta \mathrm{P}_{\nu}+\left[1-\sum_{\nu} \rho_{\nu}(s)\right] \eta(z-j) .
$$

$$
\rho_{\nu}(s)\left(z-z_{p}\right) \mathrm{P}_{\nu} \rightarrow 0, \quad z-j \rightarrow 0 \quad \text { as } t \rightarrow 0,
$$

we have

$$
\Omega(z, \bar{z}) \rightarrow 0 \text { as } t \rightarrow 0 .
$$

We investigate the derivative $\partial \Omega / \partial z$. First we observe that $\partial \rho_{v} / \partial z=\rho_{v}^{\prime}(\partial s / \partial z)$ is bounded in $S$ and that $\partial \eta / \partial z$ is bounded in $S-\sum, S_{\nu}$. Further we calculate

$$
\frac{\partial}{\partial z} \mathrm{H}_{\nu}^{-\omega_{\nu}}\left(z-z_{\nu}\right) \eta=\left(1-\omega_{\nu}\right) \mathrm{H}_{\nu}^{-\omega_{\nu} \eta}+\mathrm{H}_{\nu}^{-\omega_{\nu}}\left(z-z_{\nu}\right) \frac{\partial \eta}{\partial z} .
$$

We now use relation (4.1); because of $\left|\mathrm{H}_{\nu}\right|=1$ we get

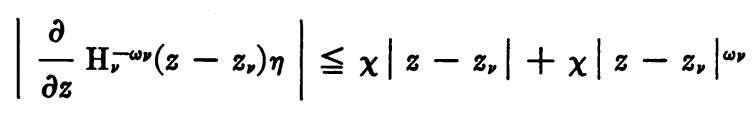

in $S_{v}$. Thus, on account of (4.3) and (4.6) we find

$$
\sum_{\nu} \mathrm{P}_{\nu} \frac{\partial}{\partial z} \rho_{\nu} \mathrm{H}_{\nu}^{-\omega_{\nu}}\left(z-z_{\nu}\right) \eta+(z-j) \frac{\partial}{\partial z}\left[1-\sum_{\nu} \rho_{\nu}\right] \eta \rightarrow 0
$$

as $t \rightarrow 0$, uniformly in $s$. 
Consequently we can choose a positive number $T_{1} \leqq T$ such that for $0<t \leqq T_{1}$

$$
\left|\frac{\partial \Omega}{\partial z}-\sum_{\nu} \rho_{\nu} \mathrm{H}_{\nu}^{-\omega_{\nu}}\left(z-z_{\nu}\right) \eta \frac{\partial \mathrm{P}_{\nu}}{\partial z}-\left[1-\sum_{\nu} \rho_{\nu}\right] \eta\left(1-\frac{\partial j}{\partial z}\right)\right| \leqq \epsilon .
$$

According to (4.4) and (4.5) this relation leads to

$$
\left|\frac{\partial \Omega}{\partial z}-\eta\right| \leqq \sum_{\nu} \rho_{\nu}\left|\frac{\sin \omega_{\nu} \pi}{\omega_{\nu} \pi}\right|+7_{\epsilon} \leqq M+\epsilon_{1},
$$

where $\epsilon_{1}=7 \epsilon$.

Let $S_{1}$ be the strip $0<t<T_{1}$ and $B_{1}^{\prime}$ the curve $t=T_{1}$; since $\Omega \rightarrow 0$ as $t \rightarrow 0$ we have the identity

$$
\iint_{S_{1}} w^{2} \frac{\overline{\partial \Omega}}{\partial z} d x d y=\frac{i}{2} \int_{B_{1^{\prime}}} w^{2} \bar{\Omega} d z
$$

From this and relation (4.8) we deduce

$$
\iint_{S_{1}} w^{2} \bar{\eta} d x d y \leqq \gamma_{1} \int_{B_{1^{\prime}}}|w|^{2}|d z|+\left(\mathbf{M}+\epsilon_{1}\right) \iint_{S_{1}}|w|^{2} d x d y
$$

where $\gamma_{1}=2 \max |\Omega|$ on $B_{1}^{\prime}$. Since $\epsilon_{1}$ is arbitrarily small we thus have proved Lemma 1 also for the case where the boundary has corners.

\section{Additional lemmas}

In proving our theorems we further make use of the following elementary lemmas.

Lemma 2. Let $D^{\prime}$ be a closed domain within $D$. Then for all functions w(z) in $\mathfrak{F}$ and all $z^{\prime}$ in $D^{\prime}$ we have

$$
\begin{aligned}
\left|w\left(z^{\prime}\right)\right|^{2} & \leqq C_{0}^{\prime} \iint_{D}|w|^{2} d x d y \\
\left|\frac{d w}{d z}\left(z^{\prime}\right)\right|^{2} & \leqq C_{1}^{\prime} \iint_{D}|w|^{2} d x d y,
\end{aligned}
$$

$C_{0}^{\prime}, C_{1}^{\prime}$ being positive constants depending on $D^{\prime}$.

We omit the proof of this well known lemma, which is an immediate consequence of the mean-value theorem and the Schwarz inequality.

Lemma 3. Let $D^{\prime}$ be a closed subdomain within $D$ and $z_{0}$ a point of $D^{\prime}$. Then for all functions $w(z)=u(z)+i v(z)$ for which 


$$
u\left(z_{0}\right)=0
$$

the inequality

$$
\left|w\left(z^{\prime}\right)\right|^{2} \leqq C^{\prime} \iint_{D} v^{2} d x d y, \quad z^{\prime} \text { in } D^{\prime},
$$

is valid, $C^{\prime}$ being a positive constant depending on $D^{\prime}$ and $z_{0}$.

We choose a finite number of points $z_{0}, z_{1}, z_{2}, \cdots, z_{j}, \cdots, z_{k}$ in $D^{\prime}$ and a positive number $R$ such that $\left|z_{j}-z_{i-1}\right|<R$, that every point $z^{\prime}$ of $D^{\prime}$ belongs to one of the circles $\left|z-z_{j}\right|<R$ and that the circles $\left|z-z_{j}\right|<3 R$ are in the interior of $D$. Now we take the relation

$$
w\left(z^{\prime}\right)-u\left(z_{j}\right)=\frac{1}{2 \pi} \int_{\left|z-z_{j}\right|=r} v(z) \frac{z+z^{\prime}-2 z_{j}}{z-z^{\prime}} \frac{d z}{z-z_{j}}
$$

which holds for $\left|z^{\prime}-z_{j}\right|<r$. We multiply by $r d r$ and integrate with respect to $r$ from $r=2 R$ to $r=3 R$ and apply the Schwarz inequality assuming $\left|z^{\prime}-z_{j}\right|<R$. Thus we obtain

$$
\left|w\left(z^{\prime}\right)-u\left(z_{j}\right)\right| \leqq 4\left(\frac{1}{5 R^{2} \pi} \iint_{2 R<\left|z-z_{j}\right|<3 R} v^{2} d x d y\right)^{1 / 2} \leqq \frac{2}{R}\left(\iint_{D} v^{2} d x d y\right)^{1 / 2}
$$

Since $u\left(z_{0}\right)=0$ and $\left|z_{j}-z_{j-1}\right|<R$, we get for every point $z^{\prime}$ of $D^{\prime}$

$$
\left|w\left(z^{\prime}\right)\right| \leqq(k+1) \frac{2}{R}\left(\iint_{D} v^{2} d x d y\right)^{1 / 2}
$$

Herewith we have proved Lemma 3.

\section{Fundamental INEQUALITIES}

We now are ready for the proof of

THEOREM 1. There exists a positive number $\Gamma$ such that the inequality

$$
\iint_{D} u^{2} d x d y \leqq \Gamma \iint_{D} v^{2} d x d y
$$

holds for all functions $w=u+i v$ in $\mathfrak{F}$ which satisfy the condition

$$
\iint_{D} u d x d y=0
$$

We first observe that it is sufficient to prove the inequality for all functions $w=u+i v$ in $\mathfrak{F}$ which vanish at a certain point $z_{0}$ in $D$; this fact follows from the relation 


$$
\iint_{D} u^{2}(z) d x d y \leqq \iint_{D}\left[u(z)-u\left(z_{0}\right)\right]^{2} d x d y,
$$

which is an immediate consequence of $\iint_{D} u d x d y=0$. Now we choose a positive number $\epsilon$ such that $M+\epsilon<1$; this is possible since $M<1$. By (2.2) of Lemma 1 we have

$$
\begin{aligned}
(1-\mathrm{M}-\epsilon) \iint_{D} u^{2} d x d y \leqq & \iint_{D-S} u^{2} d x d y+(1+\mathrm{M}+\epsilon) \iint_{S} v^{2} d x d y \\
& +\gamma \int_{B^{\prime}}|w|^{2}|d z| .
\end{aligned}
$$

We choose a point $z_{0}$ of $D-S$ and assume $u\left(z_{0}\right)=0$. Then we apply Lemma 3 to the closed domain $D^{\prime}=D-S$ and get

$$
\begin{aligned}
& (1-\mathrm{M}-\epsilon) \iint_{D} u^{2} d x d y \\
& \quad \leqq\left[C^{\prime} \iint_{D-S} d x d y+(2+\epsilon)+C^{\prime} \int_{B^{\prime}}|d z|\right] \iint_{D} v^{2} d x d y ;
\end{aligned}
$$

thus Theorem 1 is proved.

The inequality of Theorem 1 is equivalent to

$$
(\Gamma+1) \Re \iint_{D} w^{2} d x d y \leqq(\Gamma-1) \iint_{D}|w|^{2} d x d y,
$$

and the additional condition is the same as

$$
\Re \iint w d x d y=0 .
$$

Now let $w$ be a function in $\mathfrak{F}$ which satisfies the relation

$$
\iint_{D} w d x d y=0
$$

then we can choose a number $\eta$ of absolute value 1 such that

$$
\Re \iint_{D}(\eta w)^{2} d x d y=\left|\iint_{D} w^{2} d x d y\right| ;
$$

since $\Re \iint_{D} \eta w d x d y=0$ we can apply Theorem 1 to $\eta w$. On setting $\theta=(\Gamma-1) /(\Gamma+1)$ we thus obtain 
THEOREM 2. There exists a constant $\theta<1$ such that the inequality

$$
\left|\iint_{D} w^{2} d x d y\right| \leqq \theta \iint_{D}|w|^{2} d x d y
$$

holds for all functions in $\mathfrak{F}$ satisfying the condition

$$
\iint_{D} w d x d y=0 .
$$

\section{SPACE $\mathfrak{F}$ AS A HILbERT SPACE}

To prove our expansion theorem we start with the observation that the manifold $\mathfrak{F}$ of all functions $w(z)$ for which

$$
\iint_{D}|w|^{2} d x d y
$$

is finite constitutes a linear metric space. This space is either complex or real depending on whether we allow multiplication by complex or real numbers and define an inner product either by

or by

$$
\left(w_{1}, w_{2}\right)=\iint_{D} \bar{w}_{1} w_{2} d x d y
$$

$$
\left(w_{1}, w_{2}\right)=\Re \iint_{D} \bar{w}_{1} w_{2} d x d y .
$$

In either case the modulus

$$
(w, w)^{1 / 2}=\left(\iint_{D}|w|^{2} d x d y\right)^{1 / 2}
$$

has the same value. In order that

$$
\Re \iint_{D} w_{1} w_{2} d x d y
$$

become a symmetric bilinear form we choose the second definition of $\left(w_{1}, w_{2}\right)$ and thus assume $\mathfrak{F}$ to be a real space. In this case, $i w$ belongs to the space $\mathfrak{F}$ together with $w_{1}$; but this function $i w$ is orthogonal to $w$ :

$$
(w, i w)=\Re \iint_{D} \bar{w} i w d x d y=\Re i \iint_{D}|w|^{2} d x d y=0 .
$$

Now we establish

LemMa 4. The space $\mathfrak{F}$ of functions $w(z)$ is a Hilbert space. 
First we prove that $\mathfrak{F}$ is complete. Let $w^{n}(z)$ be a sequence in $\mathfrak{F}$ such that $\left(w^{m}-w^{n}, w^{m}-w^{n}\right)=\iint_{D}\left|w^{m}-w^{n}\right|^{2} d x d y \rightarrow 0$ as $m, n \rightarrow \infty$.

From the inequality (1) of Lemma 2 we deduce that $w^{m}(z)-w^{n}(z)$ converges to zero uniformly in every closed subdomain $D^{\prime}$ of $D$. From this fact it follows by a well known procedure that (i) $\iint_{D}|w|^{2} d x d y<\infty$ and therefore $w(z)$ belongs to the space $\mathfrak{F}$; and (ii)

$$
\iint_{D}\left|w^{n}-w\right|^{2} d x d y \rightarrow 0 \text { as } n \rightarrow \infty .
$$

$\mathfrak{F}$ is a subspace of the linear space $\dagger$ of all complex-valued functions $k(x, y)$, continuous in $D$, for which

$$
(k \mid k)=\iint_{D}|k|^{2} d x d y<\infty ;
$$

since, obviously, this space is separable $\mathfrak{F}$ has a like property. Therefore $\mathfrak{F}$ is a Hilbert space.

A sequence of functions $w^{1}(z), w^{2}(z), \cdots, w^{n}(z), \cdots$ in $\mathfrak{F}$ is called weakly convergent to a function $w(z)$ in $\mathfrak{F}$,

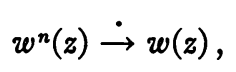

if it has the following two properties:

(i) there is a constant $M_{0}>0$ such that

(ii)

$$
\begin{gathered}
\iint_{D}\left|w^{n}\right|^{2} d x d y \leqq M_{0} ; \\
\iint_{D} \bar{W}\left(w^{n}-w\right) d x d y \rightarrow 0 \text { as } n \rightarrow \infty
\end{gathered}
$$

for each function $W(z)$ in $\mathfrak{F}$.

We have the

LEMMA 5. Let $w^{1}(z), w^{2}(z), \cdots, w^{n}(z)$ be a sequence of functions in $\mathfrak{F}$ for which

$$
\iint_{D}\left|w^{n}\right|^{2} d x d y \leqq M_{0}
$$

and which converges to a function $w_{0}(z)$ uniformly in the interior. Then $w_{0}(z)$ belongs to $\mathfrak{F}$ and $w^{n}(z)$ converges weakly to $w_{0}(z)$.

$\dagger$ Cf. Part II, §2. 
1. From $\iint_{D^{\prime}}\left|w^{n}\right|^{2} d x d y \leqq M_{0}$ we get $\iint_{D^{\prime}}\left|w_{0}\right|^{2} d x d y \leqq M_{0}$ and therefore $w_{0}(z)$ belongs to $\mathfrak{F}$.

2. Let $W(z)$ be a function of $\mathfrak{F}$. Without loss of generality, we may assume $w_{0}(z)=0$. To a given number $\epsilon>0$ we choose a subdomain $D^{\prime}$ such that $\iint_{D-D^{\prime}}|W|^{2} d x d y \leqq \epsilon^{2}$ and a number $n_{\epsilon}$ such that. $\left|w^{n}(z)\right| \leqq \epsilon$ in $D^{\prime}$ for $n \geqq n_{\epsilon}$. Then we have for $n \geqq n$.

$$
\left|\iint_{D} \bar{W} w^{n} d x d y\right| \leqq \epsilon^{2}\left[\iint_{D} d x d y \iint_{D}|W|^{2} d x d y+M\right]
$$

and therefore $\iint_{D} \bar{W} w^{n} d x d y \rightarrow 0$ as $n \rightarrow \infty$.

LEMMa 6. A sequence $S$ of functions $w^{1}(z), w^{2}(z), \ldots$ in $\mathfrak{F}$ which converges weakly to zero converges to zero uniformly in the interior of $D$.

The sequence $\subseteq$ is equicontinuous in the interior of $D$; this fact follows from (2) of Lemma 2 and $\iint_{D}\left|w^{n}\right|^{2} d x d y \leqq M_{0}$. Therefore it is sufficient to show that the sequence $\subseteq$ converges to zero at every point of $D$, the uniformity being a consequence of the equicontinuity.

Let $z_{0}$ be a point of $D$; let $\mathfrak{S}^{\prime}$ be a subsequence of $\subseteq$ which at $z_{0}$ converges to a certain value $w$. Since $\widetilde{S}^{\prime}$ is also equicontinuous, it contains a subsequence $\mathfrak{S}^{\prime \prime}$ which converges uniformly in the interior to a certain function $w_{0}(z)$. According to Lemma $5, w_{0}(z)$ belongs to $\mathfrak{F}$ and $\mathfrak{S}^{\prime \prime}$ converges weakly to $w_{0}(z)$. As $\mathfrak{S}^{\prime \prime}$ also converges weakly to zero, we have $w_{0}(\dot{z})=0$. Therefore $w=w_{0}\left(z_{0}\right)=0$. Thus $\subseteq$ converges to zero in every point of $D$ and Lemma 6 is proved.

\section{A CHARACTERISTIC VALUE PROBLEM}

In the present paragraph we discuss the form

$$
w_{1} V w_{2}=\Re \iint_{D} w_{1} w_{2} d x d y
$$

where $w_{1}(z)$ and $w_{2}(z)$ are functions in $\mathfrak{F}$; we observe that this form is (i) symmetric,

$$
w_{1} V w_{2}=w_{2} V w_{1}
$$

(ii) bilinear,

$$
w V\left(a_{1} w_{1}+a_{2} w_{2}\right)=a_{1}\left(w V w_{1}\right)+a_{2}\left(w V w_{2}\right), \quad\left(a_{1}, a_{2} \text { real }\right) ;
$$

(iii) bounded,

$$
|w V w| \leqq(w, w) .
$$


Such a form is called completely continuous, if, as $n \rightarrow \infty, w^{n} V w^{n} \rightarrow 0$ for every sequence $w^{n}(z)$ of functions in $\mathfrak{F}$ which converges weakly to zero.

An immediate consequence of Lemmas 4 and 5 is

THEOREM 3. The form $\Re \iint_{D} w^{2} d x d y$ is completely continuous if the boundary has no corners except internal cusps $(\omega=2)$.

Let $w^{n}(z)$ be a sequence which converges weakly to zero. To prove the theorem it is sufficient to show that for every number $\epsilon>0$ we can determine a number $n_{e}$ such that $\left|\Re \iint_{D}\left(w^{n}\right)^{2} d x d y\right| \leqq 3 M_{0} \in$ for $n \geqq n_{\epsilon}$. To do this, we refer to Lemma 1 and the notation there used. Let $S$ be the boundary strip of Lemma 1 corresponding to the given number $\epsilon$, let $B^{\prime}$ be the inner boundary of $S$. Then, according to Lemma 6 , there is a number $n_{\mathrm{e}}$ such that

$$
\iint_{D-S}\left|w^{n}\right|^{2} d x d y \leqq \epsilon M_{0} \quad \text { and } \quad \gamma \int_{B^{\prime}}\left|w^{n}\right|^{2}|d z| \leqq \epsilon M_{0} \quad \text { for } \quad n \geqq n_{\mathrm{e}} .
$$

Now, if we note that $M=0$, in (2.1) of Lemma 1 , we have

$$
\begin{aligned}
\left|\Re \iint\left(w^{n}\right)^{2} d x d y\right| & \leqq\left|\iint_{D-S}\left(w^{n}\right)^{2} d x d y\right|+\left|\iint_{S}\left(w^{n}\right)^{2} d x d y\right| \\
& \leqq \epsilon M_{0}+2 \epsilon M_{0}=3 \epsilon M_{0} .
\end{aligned}
$$

Now we can apply the general theory of completely continuous forms in Hilbert spaces. This theory shows that there exist two sequences of characteristic values $\mu_{1} \geqq \mu_{2} \geqq \mu_{3} \geqq \cdots \rightarrow 0$ and $\mu_{-1} \leqq \mu_{-2} \leqq \cdots \rightarrow 0$ and of corresponding characteristic functions $w_{1}(z), w_{2}(z), w_{3}(z), \cdots, w_{-1}(z), w_{-2}(z), \cdots$ satisfying the relations

$$
\left(w_{m}, w_{n}\right)= \begin{cases}1, & m=n, \\ 0, & m \neq n,\end{cases}
$$

with the following properties: Let $w(z)$ be an arbitrary function of $\mathfrak{F}$. On setting

$$
a_{n}=\left(w_{n}, w\right), \quad n= \pm 1, \pm 2, \pm 3, \cdots
$$

we have the relation

$$
\mu_{n} a_{n}=\left(w_{n} V w\right)
$$

and the developments

$$
\begin{aligned}
& (w, w)=a_{1}^{2}+a_{2}^{2}+\cdots+a_{-1}^{2}+a_{-2}^{2}+\cdots \\
& (w V w)=\mu_{1} a_{1}^{2}+\mu_{2} a_{2}^{2}+\cdots+\mu_{-1} a_{-1}^{2}+\mu_{-2} a_{-2}^{2}+\cdots .
\end{aligned}
$$

If for any value $\mu_{*}$ a function $w_{*}(z)=0$ in $\mathfrak{F}$ satisfies the characteristic 
relation $w V w_{*}=\mu_{*}\left(w, w_{*}\right)$ for all $w$ of $\mathfrak{F}$, then $\mu_{*}$ is contained among the values $\mu_{n}$ and the function $w_{*}(z)$ is a linear combination of the characteristic functions belonging to all $\mu_{n}=\mu_{*}$.

We observe that, simultaneously with $\mu_{n}$ and $w_{n}(z),-\mu_{n}$ and $i w_{n}(z)$ also satisfy the characteristic relation. For that reason we can set

$$
\mu_{-n}=-\mu_{n} \text { and } w_{-n}(z)=i w_{n}(z) .
$$

Then

$$
\iint_{D} \overline{w_{n}(z)} w_{m}(z) d x d y= \begin{cases}1, & n=m, \\ 0, & n \neq m\end{cases}
$$

and, on setting

$$
c_{n}=a_{n}-i a_{-n}=\iint_{D} \overline{w_{n}(z)} w(z) d x d y,
$$

we obtain

$$
\begin{aligned}
& \iint_{D}|w|^{2} d x d y=\left|c_{1}\right|^{2}+\left|c_{2}\right|^{2}+\cdots \\
& \Re \iint_{D} w^{2} d x d y=\Re\left[\mu_{1} c_{1}^{2}+\mu_{2} c_{2}^{2}+\cdots\right] .
\end{aligned}
$$

If we take $e^{i \pi / 4} w(z)$ instead of $w(z)$, we get

$$
\Im \iint_{D} w^{2} d x d y=\Im\left[\mu_{1} c_{1}^{2}+\mu_{2} c_{2}^{2}+\cdots\right] .
$$

We further observe the relation

$$
\begin{aligned}
\iint_{D} \mid w & -c_{1} w_{1}-c_{2} w_{2}-\cdots-\left.c_{n} w_{n}\right|^{2} d x d y \\
& =\iint_{D}|w|^{2} d x d y-\left|c_{1}\right|^{2}-\left|c_{2}\right|^{2}-\cdots-\left|c_{n}\right|^{2} \rightarrow 0 \text { and } n \rightarrow \infty,
\end{aligned}
$$

and we deduce from it, according to Lemma 2, that $c_{1} w_{1}(z)+c_{2} w_{2}(z)+\cdots$ converges to $w(z)$ uniformly in the interior. We may record these results by formulating

THEOREM 4. If the boundary $B$ has no corners except internal cusps, then there is a sequence of non-negative characteristic values

$$
\mu_{1} \geqq \mu_{2} \geqq \mu_{3} \geqq \cdots \rightarrow 0
$$

and a sequence of characteristic functions in $\mathfrak{F}$ 


$$
w_{1}(z), w_{2}(z), \cdots
$$

orthonormal in the sense

$$
\iint_{D} \bar{w}_{n} w_{m} d x d y= \begin{cases}1, & n=m, \\ 0, & n \neq m,\end{cases}
$$

which satisfy the characteristic relation

$$
\iint_{D} w_{n} w d x d y=\mu_{n} \iint_{D} \bar{w}_{n} w d x d y
$$

for every function $w(z)$ in $\mathfrak{F}$. For each function $w(z)$ in $\mathfrak{F}$ we have an expansion

$$
w(z)=c_{1} w_{1}(z)+c_{2} w_{2}(z)+\cdots
$$

converging uniformly in the interior of $D$ and for the corresponding quadratic forms

$$
\begin{aligned}
& \iint_{D}|w|^{2} d x d y=\left|c_{1}\right|^{2}+\left|c_{2}\right|^{2}+\left|c_{3}\right|^{2}+\cdots, \\
& \iint_{D} w^{2} d x d y=\mu_{1} c_{1}^{2}+\mu_{2} c_{2}^{2}+\mu_{3} c_{3}^{2}+\cdots,
\end{aligned}
$$

where

$$
c_{n}=\iint_{D} \bar{w}_{n} w d x d y
$$

Since

and the equality

$$
\left|\iint_{D} w^{2} d x d y\right| \leqq \iint_{D}|w|^{2} d x d y
$$

$$
\iint_{D} w^{2} d x d y=\iint_{D}|w|^{2} d x d y
$$

actually holds if and only if $w$ is a real constant, we may state

REMARK 1. The first characteristic value $\mu_{1}=1$, and the first characteristic function $w(z)$ is a real constant.

REMARK 2. The second characteristic value is less than $1, \mu_{2}<1$.

This inequality and the developments of $\iint_{D}|w|^{2} d x d y$ and $\iint_{D} w^{2} d x d y$ confirm Theorem 2, since relation $\iint_{D} w d x d y=0$ is equivalent to $c_{1}=0$ and $\mu_{n} \leqq \mu_{2}=\theta$ for $n=2,3,4$,

\section{CASES OF CIRCLE AND ELLIPSE}

For the circle and the ellipse the characteristic values and functions can be given explicitly. 
THEOREM 5. If $D$ is the circle $|z|<1$, then $\mu_{2}=\mu_{3}=\cdots=0$.

For every analytic function $W(z)$ which is regular in $|z|<1$ the mean value theorem gives

$$
\iint_{D} W(z) d x d y=W(0) .
$$

Since together with $w(z)$ also $w^{2}(z)$ is analytic in $D$, we have

$$
\begin{aligned}
& \iint_{D} w d x d y=w(0), \\
& \iint_{D} w^{2} d x d y=w^{2}(0) .
\end{aligned}
$$

Consequently $c_{1}=0$ is equivalent to $w(0)=0$ and for all functions with $c_{1}=0$ we have

$$
\iint_{D} w^{2} d x d y=0
$$

Theorem 6. Let $D$ be the ellipse

then

$$
\frac{x^{2}}{\cosh ^{2} \sigma}+\frac{y^{2}}{\sinh ^{2} \sigma}<1,
$$

$$
\mu_{n}=\frac{n \sinh 2 \sigma}{\sinh 2 n \sigma}, \quad n=1,2,3, \cdots
$$

and the characteristic functions are the derivatives of the Tchebycheff polynomials,

$$
w_{n}(z)=\rho_{n} \frac{\sinh (n \operatorname{arc} \cosh z)}{\sinh (\operatorname{arccosh} z)}=\rho_{n} \frac{2^{n-1}}{n} T_{n}^{\prime}(z),
$$

where

$$
\rho_{n}=\left(\frac{2 n}{\pi \sinh 2 n \sigma}\right)^{1 / 2} .
$$

These functions $w_{1}(z), w_{2}(z), w_{3}(z), \cdots$ are polynomials of degrees $0,1,2, \cdots$. The set of such polynomials is complete in the sense that every function $w(z)$ in $\mathfrak{F}$ can be approximated by a polynomial

$$
p_{n}(z)=c_{1}^{n} w_{1}(z)+c_{2}^{n} w_{2}(z)+\cdots+c_{n}^{n} w_{n}(z),
$$

so that 


$$
\iint_{D}\left|w(z)-p_{n}(z)\right|^{2} d x d y
$$

becomes arbitrarily small. $\dagger$ It remains to prove the orthogonality relation and the characteristic relation. To simplify the calculations we transform the ellipse $D$ into a rectangular domain $\Delta$ of the $(\zeta=\xi+i \eta)$-plane. We set $z=\cosh \zeta$ and $\Delta: 0 \leqq \xi<\sigma,-\pi<\eta<\pi$. Then we have

$$
\iint_{D} \bar{w}_{n} w_{m} d x d y=\rho_{n} \rho_{m} \iint_{\Delta} \cosh n \zeta \cosh m \zeta d \xi d \eta=0,
$$

if $n \neq m$. Also

$$
\begin{aligned}
\iint_{D}\left|w_{n}\right|^{2} d x d y & =\rho_{n}^{2} \iint_{\Delta}|\cosh n \zeta|^{2} d \xi d \eta \\
& =\rho_{n}^{2} \int_{-\pi}^{\pi} \int_{0}^{\sigma} \frac{1}{2} \cosh 2 n \xi d \xi d \eta \\
& =\rho_{n}^{2} \pi \frac{1}{2 n} \sinh 2 n \sigma=1, \\
\iint_{D} w_{n} w_{m} d x d y & =\rho_{n} \rho_{m} \iint_{\Delta} \frac{\sinh n \zeta \sinh m \zeta}{\sinh \zeta} \overline{\sinh \zeta} d \xi d \eta \\
& =\sum_{\alpha} \iint_{\Delta} \sinh \alpha \zeta \overline{\sinh \zeta} d \xi d \eta,
\end{aligned}
$$

where $\alpha$ runs through

$$
\alpha=m-n+1, m-n+3, \cdots, m+n-1, \text { if } m \geqq n .
$$

The integral $\iint_{\Delta} \sinh \alpha \zeta \overline{\sinh \zeta} d \xi d \eta$ vanishes except when $\alpha=1$, in which case its value is $\rho_{1}^{-2}$; this case occurs only, if $m=n$; therefore we have

$$
\iint_{D} w_{n} w_{m} d x d y= \begin{cases}0, & n \neq m, \\ \rho_{n}{ }^{2} / \rho_{1}{ }^{2}=\mu_{n}, & n=m .\end{cases}
$$

10. GENERAL CASE OF BOUNDARY WITH CORNERS

Since the form $\Re \iint_{D} w^{2} d x d y$ is symmetric and bounded, the general theory of spectra $\ddagger$ is applicable also when this form is not completely continuous,

$\dagger$ L. Bieberbach, Zur Theorie und Praxis der konformen Abbildung, Rendiconti del Circolo Matematico di Palermo, vol. 38 (1914), p. 98.

T. Carleman, Über die Approximation analytischer Funktionen durch lineare Aggregate von vorgegebenen Potenzen, Arkiv för Matematik, Astronomi och Fysik, vol. 17, No. 9 (1922).

For a more detailed reference, see J. L. Walsh, Approximation by Polynomials in the Complex Domain, Mémorial des Sciences Mathématiques, No. 73, 1935, p. 61.

$\ddagger$ Cf. M. H. Stone, Linear Transformations in Hilbert Space and their Applications to Analysis, American Mathematical Society Colloquium Publications, vol. 15, 1932. 
in which case it has a continuous spectrum. We use the notion of limit spectrum (Häufungsspektrum) in the sense of Weyl; $\dagger$ this closed set of values $\mu$ consists of all points of the continuous spectrum, of the limit points of the characteristic values and of all characteristic values of infinite order. In every closed interval outside of the limit spectrum there are only a finite number of characteristic values and these have a finite order. The limit spectrum consists of the single point $\mu=0$ if and only if the form is completely continuous. Now we can prove

Theorem 7. The l.u.b. $\bar{\mu}$ and the g.l.b. $\underline{\mu}$ of the limit spectrum of the form $\Re \iint_{D} w^{2} d x d y$ are precisely $\mathrm{M}$ and $-\mathrm{M}$ respectively. Therefore Theorem 4 is valid, if and only if $\mathrm{M}=0$; that is to say, if the boundary has no corner except interior cusps.

First we show that the limit spectrum is contained in the interval $-\mathbf{M} \leqq \mu \leqq M$. From Lemma 1 we derive the inequality

$$
\begin{aligned}
\Re \iint_{D} w^{2} d x d y-\iint_{D-S}|w|^{2} d x d y-\gamma \int_{B^{\prime}}|w|^{2}|d z| & \\
& \leqq(\mathrm{M}+\epsilon) \iint_{D}|w|^{2} d x d y
\end{aligned}
$$

which shows that the l.u.b. of the whole spectrum and therefore also the l.u.b. of the limit spectrum of the form of the left-hand member is not greater than $M+\epsilon$. Now we refer to the fundamental theorem of Weyl $\ddagger$ to the effect that the limit spectrum of a form remains unaltered whenever the form is changed by adding a completely continuous form. Now, according to Lemma 6 , the forms $\iint_{D-S}|w|^{2} d x d y$ and $\gamma \int_{B^{\prime}}|w|^{2}|d z|$ are completely continuous and therefore the l.u.b. of the limit spectrum of $\Re \iint_{D} w^{2} d x d y$ is not greater than $\mathbf{M}$, since $\epsilon$ is arbitrarily small. The same reasoning shows that the g.l.b. is not less than $-M$. Thus we have proved that $-\mathbf{M} \leqq \underline{\mu} \leqq \bar{\mu} \leqq M$.

We now prove that $\bar{\mu} \geqq M$. According to a remark of $H$. Weyl this value has the following property: Whenever there is a sequence of functions $w(z)$ in $\mathfrak{F}$ weakly convergent to zero for which

then

$$
\Re \iint_{D} w^{2} d x d y: \iint_{D}|w|^{2} d x d y \rightarrow \mu,
$$

$$
\mu \leqq \bar{\mu} .
$$

† H. Weyl, Über beschränkte quadratische Formen, deren Differenz vollstetig ist, Rendiconti del Circolo Matematico di Palermo, vol. 27 (1909), pp. 373-392.

$\ddagger$ Loc. cit. 
Let $z=0$ be a corner of the boundary $B$ with an angle $\omega$ and with $|\sin \omega \pi / \omega \pi|=\mathrm{M}$. We set $z=r e^{i \vartheta}$. We represent the two branches of $B$ in the neighborhood of $z=0$ by $\vartheta=\vartheta_{+}(r)$ and $\vartheta=\vartheta_{-}(r)$ and choose a number $R>0$ such that the domain

$$
D_{R}: \quad \vartheta_{-}(r)<\vartheta<\vartheta_{+}(r), \quad 0<r<R,
$$

is contained in $D$.

We may assume $\vartheta_{+}(0)=\omega \pi / 2, \vartheta_{-}(0)=-\omega \pi / 2$. Then we set $\eta=1$ if $\sin \omega \pi>0, \eta=i$ if $\sin \omega \pi<0$ and choose the sequence

$$
w_{\alpha}(z)=\eta(2 \alpha)^{1 / 2} z^{\alpha-1}, \quad \alpha \rightarrow 0 .
$$

We calculate $\iint_{D}\left|w_{\alpha}\right|^{2} d x d y$ and $\iint_{D} w_{\alpha}^{2} d x d y$. On setting

$$
\begin{gathered}
\vartheta_{+}(r)-\vartheta_{-}(r)=\omega \pi+r \theta(r), \\
\int_{\vartheta_{-}(r)}^{\vartheta_{+}(r)} e^{(2 \alpha-2) i \vartheta} d \vartheta=\sin \omega \pi+r \phi(r),
\end{gathered}
$$

we find that $|\theta(r)|$ and $|\phi(r)|$ are bounded for $0<r<R$. Hence, as $\alpha \rightarrow 0$, we have

$$
\begin{aligned}
& \iint_{D}\left|w_{\alpha}\right|^{2} d x d y=\omega \pi R^{2 \alpha}+2 \alpha \int_{0}^{R} r^{2 \alpha} \theta(r) d r+2 \alpha \iint_{D-D_{R}} r^{2 \alpha-2} d x d y \rightarrow \omega \pi \\
& \iint_{D} w_{\alpha}^{2} d x d y=|\sin \omega \pi| R^{2 \alpha}+2 \alpha \eta^{2} \int_{0}^{R} r^{2 \alpha} \phi(r) d r+2 \alpha \iint_{D-D_{R}} z^{2 \alpha-2} d x d y \rightarrow|\sin \omega \pi|,
\end{aligned}
$$

and therefore

$$
\iint_{D} w_{\alpha}^{2} d x d y: \iint_{D}\left|w_{\alpha}\right|^{2} d x d y \rightarrow\left|\frac{\sin \omega \pi}{\omega \pi}\right|=\mathrm{M} .
$$

According to Lemma 5, the sequence $w_{\alpha}(z)$ converges weakly to zero, as $\alpha \rightarrow 0$. Thus we obtain $M \leqq \bar{\mu}$. If we take the sequence $w_{\alpha}(z)=i \eta(2 \alpha)^{1 / 2} z^{\alpha-1}$ we get $-M \geqq \mu$. So we have proved Theorem 7 .

Remark. In the case where the boundary $B$ has an external cusp which was excluded hitherto it can happen that the limit spectrum reaches the points $\mu=1$ and $\mu=-1$ and that, consequently, the inequality theorem does not hold.

We assume that the boundary $B$ has an internal cusp at the point $z=0$. On introducing the functions $\vartheta=\vartheta_{+}(r), \vartheta=\vartheta_{-}(r)$ and the domain $D_{R}$ as before we assume that 


$$
\vartheta_{+}(r)=\frac{\kappa}{2} r+r^{2} \theta_{+}(r), \quad \vartheta_{-}(r)=-\frac{\kappa}{2} r+r^{2} \theta_{-}(r),
$$

where $\kappa \neq 0$ and $\theta_{+}, \theta_{-}$are bounded for $0<r<R$. Then we choose the sequence

$$
w_{\alpha}(z)=(2 \alpha)^{1 / 2} z^{\alpha-3 / 2} .
$$

On setting

$$
\begin{gathered}
\vartheta_{+}(r)-\vartheta_{-}(r)=\kappa r+r^{2} \theta(r), \\
\int_{\vartheta_{-}(r)}^{\vartheta_{+}(r)} e^{(2 \alpha-3) i \vartheta} d \vartheta=\kappa r+r^{2} \phi(r),
\end{gathered}
$$

we find that $\theta(r)$ and $\phi(r)$ are bounded for $0<r<R$. Hence, as $\alpha \rightarrow 0$, we have

$$
\begin{gathered}
\iint_{D}\left|w_{\alpha}\right|^{2} d x d y=\kappa R^{2 \alpha}+2 \alpha \int_{0}^{R} r^{2 \alpha} \theta(r) d r+2 \alpha \iint_{D-D_{R}} r^{2 \alpha-3} d x d y \rightarrow \kappa, \\
\iint_{D}|w|^{2} d x d y=\kappa R^{2}+2 \alpha \int_{0}^{R} r^{2 \alpha} \phi(r) d r+2 \alpha \iint_{D-D_{R}} r^{2 \alpha-3} d x d y \rightarrow \kappa,
\end{gathered}
$$

and, therefore,

$$
\iint_{D} w_{\alpha}^{2} d x d y: \iint_{D}\left|w_{\alpha}\right|^{2} d x d y \rightarrow 1 .
$$

Since $w_{\alpha}$ tends weakly to zero this relation yields $\bar{\mu}=1$.

Part II. The case of two functions of two variables

This second part is concerned with complex-valued functions $k(x, y)$ of two variables $x, y$, defined in an open domain $D$. The manifold of all such functions $k(x, y)$ for which the integral

$$
(k \mid k)=\frac{1}{2} \iint_{D}\left\{\left|\frac{\partial k}{\partial x}\right|^{2}+\left|\frac{\partial k}{\partial y}\right|^{2}\right\} d x d y
$$

is finite forms a Hilbert space $\Re$.

I consider several subspaces of $\AA$ and investigate their relations. By means of the projectors of these subspaces I can represent the operator which corresponds to the quadratic form treated in Part I.

The inequality and the expansion theorem which $I$ have established for this form can be employed here. Under the same assumption regarding the boundary, I obtain an inequality and expansions for the functions $k$ of the space $\Omega$. This inequality statement is that there exists a positive number $\sigma=\sigma_{D}$ such that 


$$
\sigma(k \mid k) \leqq \iint_{D}\left(\Re \frac{\partial k}{\partial z}\right)^{2} d x d y+\iint_{D}\left|\frac{\partial k}{\partial z}\right|^{2} d x d y
$$

for all functions $k$ in $\Re$ which satisfy the relation $\Im \iint_{D}(\partial k / \partial z) d x d y=0$. Finally I show that this inequality $\dagger$ plays a decisive part in the theory of equilibrium and vibration of an elastic plate.

\section{The Relative SPeCtrum OF TWO SUBSPaCES}

1.1. The smallest angle between two spaces. At first we make some remarks on the relative spectrum of two subspaces of a Hilbert space which we will apply to our question in functional spaces.

Let $\mathfrak{S}$ be a real Hilbert space of elements $h$ with theinner product $\left(h_{1}, h_{2}\right)$. Let $\mathfrak{F}$ and $\mathfrak{Q}$ be two closed (linear) subspaces of $\mathfrak{S}$ with the projectors $F$ and $Q$; the spaces of all elements in $\mathfrak{E}$ which are orthogonal to $\mathfrak{F}$ and $\mathfrak{Q}$ respectively are denoted by $B$ and $\mathfrak{B}$ with projectors $G$ and $P$ : So we have

$$
F+G=1, \quad F G=0, \quad P+Q=1, \quad P Q=0 .
$$

We denote by $\mathfrak{E}^{\prime}, \mathfrak{F}^{\prime}, \mathfrak{S}^{\prime}, \mathfrak{P}^{\prime}, \mathfrak{Q}^{\prime}$ the subspaces of $\mathfrak{W}, \mathfrak{F}, \mathfrak{S}, \mathfrak{B}, \mathfrak{Q}$ which are orthogonal to the four section spaces $\mathfrak{Q F}, \mathfrak{B F}, \mathfrak{Q} \mathfrak{B}, \mathfrak{B H}$; so we have $\mathfrak{S}^{\prime}=\mathfrak{B}^{\prime} \oplus \mathfrak{Q}^{\prime}=\mathfrak{F}^{\prime} \oplus \mathfrak{S}^{\prime}, \mathfrak{W}=\mathfrak{S}^{\prime} \oplus \mathfrak{Q} \mathfrak{F} \oplus \mathfrak{B F} \oplus \mathfrak{Q} \oplus \oplus \mathfrak{B S}$.

We introduce the largest non-negative number $\tau_{0} \leqq \pi / 2$ such that for all elements $f$ in $\mathfrak{F}, q$ in $\mathfrak{Q}$ which both are orthogonal to the section $\mathfrak{Q} \mathfrak{F}$ the relation

$$
(f, q)^{2} \leqq \cos ^{2} \tau_{0}(f, f)(q, q)
$$

is valid, and we call it the "smallest angle between the spaces $\mathfrak{Q}$ and $\mathfrak{F}$." The inequality (1.1) is equivalent to each of the following four inequalities:

$(Q f, Q f) \leqq \cos ^{2} \tau_{0}(f, f) ;$

$$
(P f, P f) \geqq \sin ^{2} \tau_{0}(f, f) \text {; }
$$

$$
(F q, F q) \leqq \cos ^{2} \tau_{0}(q, q) \text {; }
$$

$$
(G q, G q) \geqq \sin ^{2} \tau_{0}(q, q) .
$$

We show this for $(1.2)_{Q}$ : from (1.1) we get

$$
(Q f, Q f)^{2}=(f, Q f)^{2} \leqq \cos ^{2} \tau_{0}(f, f)(Q f, Q f)
$$

and thus $(1.2)_{Q}$; and from $(1.2)_{Q}$ we get

$\dagger$ It is the analogue of the inequality of A. Korn for functions of three variables. The expansion theorem is related to those of $\mathrm{E}$. and F. Cosserat.

Cf. A. Korn, Über einige Ungleichungen, welche in der Theorie der elastischen und elektrischen Schwingungen eine Rolle spielen, Bulletin de l'Académie des Sciences de Cracovie, 1909, vol. 2, pp. 705-724, and literature indicated therein. 


$$
(f, q)^{2}=(Q f, q)^{2} \leqq(Q f, Q f)(q, q) \leqq \cos ^{2} \tau_{0}(f, f)(q, q)
$$

and thus (1.1).

Remark. Every subspace of finite dimension has a positive smallest angle with respect to every other space in which it is not contained.

We have the following

THEOREM 1.1. If the smallest angle $\tau_{0}$ between the spaces $\mathfrak{Q}$ and $\mathfrak{Y}$ is positive, then there is a constant $\rho$ such that

$$
\rho(h, h) \leqq(h, P h)+(h, G h)
$$

for all elements $h$ in $\mathfrak{S}$ which are orthogonal to $\mathfrak{Q} \mathfrak{F}$.

For every such element $h$ can be written in the form

$$
h=f+q+j,
$$

where the elements $f$ in $\mathfrak{F}, q$ in $\mathfrak{Q}, j$ in $\mathfrak{B} \mathfrak{S}$ are orthogonal to $\mathfrak{Q} \mathfrak{F}$. Then we have

$$
\begin{aligned}
(h, h) & =(f, f)+2(f, q)+(q, q)+(j, j) \\
& \leqq(f, f)+2 \cos \tau_{0}[(f, f)(q, q)]^{1 / 2}+(q, q)+(j, j) \\
& \leqq\left(1+\cos \tau_{0}\right)[(f, f)+(q, q)]+(j, j)
\end{aligned}
$$

and

$$
\begin{aligned}
(h, P h)+(h, G h) & =(f, P f)+(q, G q)+2(j, j) \\
& \geqq \sin ^{2} \tau_{0}[(f, f)+(q, q)]+2(j, j) .
\end{aligned}
$$

Thus Theorem 1.1 is true with $\rho=1-\cos \tau_{0}$.

1.2. The spectrum of the operator $F Q F$. Now. we discuss the symmetric operator $F Q F$. It transforms every element $f$ in $\mathfrak{F}$ into an element $F Q F f$ in $\mathfrak{F}$ and we have

$$
0 \leqq(f, F Q F f) \leqq(f, f) .
$$

The operator $F Q F$, considered an operator in $\mathfrak{F}$, has a spectral resolution. For the sake of simplicity we assume that the spectrum is a pure point spectrum. A characteristic value $\kappa$ is a real number to which there are elements $f_{k} \neq 0$ in $\mathfrak{F}$ such that

$$
F Q F f_{k}=\kappa f_{k} .
$$

The space of all such characteristic elements $f_{\kappa}$ may be designated by $\left\{f_{\kappa}\right\}$; the characteristic spaces $\left\{f_{k}\right\}$ for different values $\kappa$ are orthogonal and all these spaces span the whole space $\mathfrak{F}: \sum_{\kappa}\left\{f_{k}\right\}=\mathfrak{F}$. The characteristic values $\kappa$ 
satisfy the relation $0 \leqq \kappa \leqq 1$. The characteristic spaces of $\kappa=1$ and $\kappa=0$ are the sections of $\mathfrak{Q}$ and $\mathfrak{P}$ respectively with $\mathfrak{F}$ :

$$
\left\{f_{1}\right\}=\mathfrak{Q F}, \quad\left\{f_{0}\right\}=\mathfrak{B F} .
$$

If we wish to exclude the values $\kappa=0$ and $\kappa=1$ we write $\sum^{\prime}$ instead of $\sum$. So we have $\sum_{k}^{\prime}\left\{f_{k}\right\}=\mathfrak{F}^{\prime}$. Let $f_{k}$ be such a characteristic element. Then the element $q_{\kappa}=Q f_{k}$ has the properties

$$
\left(q_{k}, q_{k}\right)=\left(q_{k} ; f_{k}\right)=\kappa\left(f_{k}, f_{k}\right) \text {. }
$$

Therefore, if $\tau$ is the angle between $q_{\kappa}$ and $f_{\kappa}$ we have

$$
\kappa=\cos ^{2} \tau \text {. }
$$

The g.l.b. of all such numbers $\tau \neq 0$ is the smallest angle $\tau_{0}$ between the spaces $\mathfrak{Q}$ and $\mathfrak{F}$ and if $\tau_{1}$ is the l.u.b. of all such numbers $\tau \neq \pi / 2$, then $\pi / 2-\tau_{1}$ is ; smallest angle between the spaces $\mathfrak{B}$ and $\mathfrak{F}$. The manifold which is spanned by the characteristic elements $f_{k}$ and by. $q_{k}=Q f_{k}$, provided that $\kappa \neq 0$ and $\kappa \neq 1$, may be denoted by $\left\{f_{k}, q_{k}\right\}$. The dimension of $\left\{f_{k}, q_{k}\right\}$ is twice the multiplicity of $\left\{f_{k}\right\}$. The subspaces $\left\{f_{\kappa}, q_{k}\right\}$ for different values $\kappa$ are orthogonal to each other, to $\mathfrak{P F}$, and to $\mathfrak{Q} F$. This fact follows from

$$
\kappa^{\prime}\left(f_{k}, f_{k^{\prime}}\right)=\left(f_{k}, q_{k^{\prime}}\right)=\left(q_{k}, q_{k^{\prime}}\right)=\left(q_{k}, f_{k^{\prime}}\right)=\kappa\left(f_{k}, f_{\kappa^{\prime}}\right) .
$$

These spaces are also orthogonal to the sections $\mathfrak{O M S}$ and $\mathfrak{P}(S)$; and we have

THEOREM 1.2. The spaces $\left\{f_{k}, q_{k}\right\}$ belonging to all characteristic values $\kappa \neq 0, \neq 1$ of the operator $F Q F$ in $\mathfrak{F}$ span the whole space $\mathfrak{S}^{\prime}: \mathfrak{S}^{\prime}=\sum_{\kappa}^{\prime}\left\{f_{\kappa}, q_{\kappa}\right\}$.

Let $h$ be an element of $\mathfrak{S}^{\prime}$ which is orthogonal to all manifolds $\left\{f_{\kappa}, q_{k}\right\}$, $(\kappa \neq 0, \neq 1)$. Then $h$ is orthogonal to all $\left\{f_{\kappa}\right\}$. Since these elements $f_{\kappa}$ span the whole space $\mathfrak{F}$, the element $h$ is orthogonal to $\mathfrak{F}$; that means $h$ belongs to $\mathbb{B S}^{\prime}$. Further, the element $h$ is orthogonal to all elements $q_{\kappa}=Q f_{\kappa}$ and, consequently, the element $Q h$ of $\mathfrak{Q}^{\prime}$ is orthogonal to $\mathfrak{F}^{\prime}$; that means: $Q h$ belongs to $\mathcal{B F}^{\prime}$ and, since $\mathfrak{Q}^{\prime} \mathfrak{S}^{\prime}=0$ we have $Q h=0$. Therefore $h$ belongs to $\mathfrak{B}^{\prime}$; but, since $\mathfrak{B}^{\prime} \mathfrak{S}^{\prime}=0$, we have $h=0$. Thus Theorem 1.2 is proved.

1.3. The spectrum of the operator $a P+b G$. We investigate the operator

$$
a P+b G
$$

where $a \neq 0$ and $b \neq 0$ are given numbers. Since this operator is symmetric and bounded it has a spectral resolution. We can express the characteristic values $\lambda$ of this operator by the characteristic values $\kappa=\cos ^{2} \tau$ of the operator $F Q F$ in $\mathfrak{F}$. To every such value we determine the solutions $\lambda_{x}$ of the quadratic equation 


$$
\left(\lambda_{\kappa}-a\right)\left(\lambda_{x}-b\right)=a b \kappa,
$$

namely,

$$
\begin{aligned}
\lambda_{k}+ & =\frac{a+b}{2}+\left[\left(\frac{a-b}{2}\right)^{2}+a b \kappa\right]^{1 / 2}=\frac{a+b}{2}+\left[\left(\frac{a+b}{2}\right)^{2}-a b \sin ^{2} \tau\right]^{1 / 2} \\
& =\frac{a+b}{2}-\left[\left(\frac{a-b}{2}\right)^{2}+a b \kappa\right]^{1 / 2}=\frac{a+b}{2}-\left[\left(\frac{a+b}{2}\right)^{2}-a b \sin ^{2} \tau\right]^{1 / 2} .
\end{aligned}
$$

If $\kappa=0$ we have $\lambda_{0}^{+}=\max (a, b), \lambda_{0}^{-}=\min (a, b)$; if $\kappa=1, \lambda_{1}^{+}=\max (a+b, 0)$, $\lambda_{1}^{-}=\min (a+b, 0)$. We have the

THEOREM 1.3. The values $\lambda=\lambda_{\star}^{ \pm}$, for every $\kappa \neq 0, \neq 1$, are characteristic values of the operator $a P+b G$; their characteristic functions are

$$
h_{k}^{ \pm}=\left(\lambda_{k}^{ \pm}-b\right) f_{k}-a q_{k} .
$$

The characteristic spaces of the values $\lambda=a$ and $\lambda=b$ are $\mathfrak{B F}$ and $\mathfrak{Q} \mathbb{S}$ respectively and the characteristic spaces of the values $\lambda=a+b$ and $\lambda=0$ are $\mathfrak{B ( S )}$ and $\mathfrak{Q} \mathfrak{F}$ respectively. The other characteristic functions span the whole space $\mathfrak{W}^{\prime}$ :

$$
B^{\prime}=\sum_{\alpha}^{\prime}\left\{h_{k}^{+}\right\}+\sum_{\kappa}^{\prime}\left\{h_{\alpha}^{-}\right\} \text {. }
$$

A simple calculation shows that the elements $h_{k}^{ \pm}$and the four section spaces are characteristic. Since

$$
\begin{gathered}
\lambda_{k}^{+}-\lambda_{k}^{-} \neq 0 \text { if } \kappa \neq 0, \neq 1, \text { we have } \\
f_{k}=\frac{h^{+}-h^{-}}{\lambda_{k}^{+}-\lambda_{k}^{-}}, \quad q_{k}=\frac{1}{a} \frac{\left(\lambda_{k}^{+}-b\right) h_{k}^{+}-\left(\lambda_{k}^{-}-b\right) h_{k}^{-}}{\lambda_{k}^{+}-\lambda_{k}^{-}}
\end{gathered}
$$

and

$$
\left\{h_{k}^{+}\right\} \oplus\left\{h_{k}^{-}\right\}=\left\{f_{k}, q_{k}\right\}, \quad \kappa \neq 0 \neq 1 .
$$

Therefore relation (1.3) follows from Theorem 1.2 and Theorem 1.3 is proved

We remark that the spectrum is contained within the two closed intervals $\left[\lambda_{0}^{-}, \lambda_{1}^{-}\right]$and $\left[\lambda_{0}^{+}, \lambda_{1}^{+}\right]$, which have a common point only if $a+b=0$ or $a=b$. Every limit point $\kappa_{\infty}$ of the $\kappa$-spectrum corresponds to two limit points $\lambda_{\infty}{ }^{+}$ and $\lambda_{\infty}{ }^{-}$of the $\lambda$-spectrum (except when $a=b, \kappa_{\infty}=0$ or $a+b=0, \kappa_{\infty}=1$ ).

If the spaces $\mathfrak{Q}$ and $\mathfrak{F}$ have a positive smallest angle $\tau_{0}>0$, then there is a constant $\rho>0$ such that $\rho(h, h) \leqq a(h, P h)+b(h, Q h)$, if $a>0, b>0$ for all $h \perp \mathfrak{O F}$. (The largest possible number $\rho$ is exactly

$$
\rho_{0}=\frac{a+b}{2}-\left[\left(\frac{a-b}{2}\right)^{2}+a b \cos ^{2} \tau_{0}\right]^{1 / 2}
$$




$$
=\frac{a b \sin ^{2} \tau_{0}}{\frac{a+b}{2}+\left[\left(\frac{a-b}{2}\right)^{2}+a b \cos ^{2} \tau_{0}\right]^{1 / 2}} .
$$

Cf. Theorem 1.1 where $a=b=1, \rho_{0}=1-\cos \tau_{0}$.)

In the same way we can treat the spectral problem of the operator $a P+b G$ with respect to a different unit-form, for example,

$$
\alpha\left(h_{1}, F h_{2}\right)+\beta\left(h_{1}, G h_{2}\right), \quad \alpha \geqq 0, \quad \beta \geqq 0 .
$$

In this case also every $\kappa$ corresponds to two characteristic values $\lambda$, the solutions of the quadratic equation

$$
(\lambda \alpha-a)(\lambda \beta-b)+(\lambda(\beta-\alpha)-b) a_{\kappa}=0,
$$

and the characteristic elements

$$
h_{k}=(\lambda \beta-b) f_{k}-a q_{k} .
$$

The section spaces $\mathfrak{B F}, \mathfrak{O G}, \mathfrak{B G}, \mathfrak{O F}$ belong to the characteristic values

$$
\frac{a}{\alpha}, \frac{b}{\beta} ; \frac{a+b}{\beta}, 0 \text {. }
$$

\section{THE SPACE $\Omega$}

2.1. The space $\Re$ in general. Let $D$ be an open domain in the $z$-plane. Let $k=k_{x}+i k_{y}$ be a complex-valued function of $x$ and $y$ defined in $D$ and having derivatives

$$
\frac{\partial k}{\partial x}, \frac{\partial k}{\partial y}
$$

with respect to $x$ and $y$ which are $L^{2}$-integrable over any subdomain $D^{*}$ of $D$

$$
(k \mid k)_{D^{*}}=\frac{1}{2} \iint_{D^{*}}\left\{\left|\frac{\partial k}{\partial x}\right|^{2}+\left|\frac{\partial k}{\partial y}\right|^{2}\right\} d x d y<\infty .
$$

Considering $k$ as a function of $z=x+i y$ and $\bar{z}=x-i y$ we have the relation

$$
(k \mid k)_{D^{*}}=\iint_{D^{*}}\left\{\left|\frac{\partial k}{\partial z}\right|^{2}+\left|\frac{\partial k}{\partial \bar{z}}\right|^{2}\right\} d x d y,
$$

because of

$$
2 \frac{\partial}{\partial z}=\frac{\partial}{\partial x}-i \frac{\partial}{\partial y}, \quad 2 \frac{\partial}{\partial z}=\frac{\partial}{\partial x}+i \frac{\partial}{\partial y} .
$$


In case of a multiply-connected domain $D$ we admit many-valued functions provided that they have singled-valued derivatives $\partial k / \partial x, \partial k / \partial y$.

We denote by $\Re$ the manifold of all functions $k(x, y)$ of this kind for which the integral

$$
(k \mid k)=\iint_{D}\left\{\left|\frac{\partial k}{\partial z}\right|^{2}+\left|\frac{\partial k}{\partial \bar{z}}\right|^{2}\right\} d x d y
$$

is finite; $\Re$ is a linear space. Two functions $k, k^{*}$ of $\Re$ are "equivalent" if $\left(k^{*}-k \mid k^{*}-k\right)=0 . \dagger$ The manifold of all functions of $\Omega$ which are equivalent to each other corresponds to one element of the space $\Omega$. So, e.g., the function $k(x, y)=$ const. corresponds to the element zero.

In the space $\Omega$ we define the inner product

$$
\left(k_{1} \mid k_{2}\right)=\Re \iint_{D}\left\{\frac{\overline{\partial k_{1}}}{\partial z} \frac{\partial k_{2}}{\partial z}+\frac{\partial \bar{k}_{1}}{\partial z} \frac{\partial k_{2}}{\partial \bar{z}}\right\} d x d y .
$$

With respect to this metric the space $\Omega$ is a real one; but to every element $k=k_{x}+i k_{y}$ of $\Re$ the operations $\Re k=k_{x}, \Im k=k_{y}, i k=k_{y}-i k_{x}, \bar{k}=k_{x}-i k_{y}$ are feasible. Corresponding to Fischer's form of the theorem of F. Riesz and E. Fischer we have the important fact:

THEOREM 2.1. The space $\Omega$ is complete.

2.2. The subspace $\mathfrak{F}$. We introduce the subspace $\mathfrak{F}$ of all elements $f$ in $\Re$ for which $\iint_{D}|\partial f / \partial \bar{z}|^{2} d x d y=0$; to these elements there correspond functions $f$ with continuous derivatives for which $\partial f / \partial \bar{z}=0$, that is to say, which are analytic functions of $z$. In the following we assume the function $f$ in $\mathfrak{F}$ to be analytic. Every function $f$ of this space $\mathfrak{F}$ can also be represented by the derivative $w=d f / d z$ and we have

$$
\left(f_{1} \mid f_{2}\right)=\Re \iint_{D} \bar{w}_{1} w_{2} d x d y .
$$

Therefore the space $\mathfrak{F}$ can be identified with the space of analytic functions $w(z)$ dealt with in Part I. As we proved there, we have

$\dagger$ For further application we need the obvious

LEMMA 2.1. Two functions $k, k^{*}$ are equivalent if

$$
\iint_{D}\left|k^{*}-k\right|^{2} d x d y=0
$$

$\ddagger$ For the proof use, e.g., the methods of G. Fubini, $I l$ principio di minimo e $i$ teoremi di esistenza per $i$ problemi al contorno relativi alle equazioni alle derivate parziali di ordine pari, Rendiconti del Circolo Matematico di Palermo, vol. 23 (1907), pp. 9-11. 
TheOrem 2.2. The space $\mathfrak{F}$ is complete.

The space $\mathfrak{F}$ of all functions $\bar{f}$ in $\Re$ for which

$$
\frac{\partial \bar{f}}{\partial z}=0
$$

consists of the conjugates of all functions $f$ in $\mathfrak{F}$. We have

$$
\left(\bar{f}_{1} \mid \bar{f}_{2}\right)=\Re \iint_{D} \frac{d f_{1}}{d \bar{z}} \frac{d \bar{f}_{2}}{d z} d x d y .
$$

From Theorem 2.2 we get:

ThEOREM 2.3. The space $\overline{\mathfrak{F}}$ is complete. $\dagger$

Since for every function $f_{1}$ in $\mathfrak{F}, \bar{f}_{2}$ in $\overline{\mathfrak{F}}$

we have

$$
\left(f_{1} \mid \bar{f}_{2}\right)=0 \text {, }
$$

THEOREM 2.4. The spaces $\mathfrak{F}$ and $\overline{\mathfrak{F}}$ are orthogonal to each other.

A function $k(x ; y)$ is called a potential function if it has continuous second derivatives satisfying the relation $\partial^{2} k / \partial z \partial \bar{z}=0$. Such a function $k(x, y)$ can be written in the form $k(x, y)=f(z)+\overline{f^{*}(z)}$, where $f(z), f^{*}(z)$ are analytic in $z$; together with $k$ also $f$ and $f^{*}$ belong to $\mathfrak{F}$. Thus we get

THEOREM 2.5. The space $\mathfrak{F} \oplus \overline{\mathfrak{F}}$ consists of the potential functions in $\Omega$.

2.3. The subspace $\$$. We introduce the subspace $\dot{\xi}$. of elements $g$ in $\Re$ which are equivalent to functions $g$ in $\Omega$ which vanish identically in a boundary strip. We denote by $(S)$ the closure of $\dot{\xi}$. That is to say, $\&$ consists of all functions $g$ in $\Re$ for which there are functions $\dot{g}$ in $\dot{S}$ such that $(\dot{g}-g \mid \dot{g}-g)$ is arbitrarily small. $\neq$ This definition contains the

THEOREM 2.6. The space \& is complete.

We establish the following basic identity:

$$
(k \mid g)=2 \Re \iint_{D} \frac{\overline{\partial k}}{\partial z} \frac{\partial g}{\partial z} d x d y=2 \Re \iint_{D} \frac{\partial \bar{k}}{\partial z} \frac{\partial g}{\partial \bar{z}} d x d y
$$

for all $k$ in $\Omega, g$ in $\$$.

$\dagger$ This theorem is related to that of S. Zaremba. Cf. S. Zaremba, Sur un problème toujours possible, comprenant, a titre de cas particulier, le problème de Dirichlet et celui de Neumann, Journal de Mathématiques, sér. 9, vol. 6 (1927), pp. 127-163; O. Nikodym, Sur un théorème de M. S. Zaremba concernant les fonctions harmoniques, Journal de Mathématiques, sér. 9, vol. 12 (1933), pp. 95-109, and Sur le principe du minimum, Mathematica Cluj, vol. 9 (1936), p. 123.

$\ddagger$ Under simple assumptions regarding the boundary it would be possible to give a direct definition of $(B)$ by a boundary condition. 
To prove it let $\dot{g}$ be a function of $\dot{\xi} \xi$ which vanishes in a boundary strip $S$. In $D$ we take a subdomain $D^{*}$ with rectifiable boundary $B^{*}$ contained in $S$. If $k$ is in $\Omega$, there exists a function $k^{*}(x, y)$ which is defined in $D^{*}+B^{*}$, has continuous second derivatives, and approximates $k$ in the sense that $\left(k^{*}-k \mid k^{*}-k\right)_{D^{*}}$ is small. The equations

$$
\begin{aligned}
\iint_{D^{*}} \frac{\overline{\partial k^{*}}}{\partial z} \frac{\partial \dot{g}}{\partial z} d x d y-\iint_{D^{*}} \frac{\partial \overline{k^{*}}}{\partial z} \frac{\partial \dot{g}}{\partial \bar{z}} d x d y \\
=\frac{i}{2} \int_{B^{*}} \frac{\overline{\partial k^{*}}}{\partial \bar{z}} d \bar{z}+\frac{i}{2} \int_{B^{*}} \frac{\partial \overline{k^{*}}}{\partial z} \dot{g} d \bar{z}=0
\end{aligned}
$$

imply that, for all functions $k$ in $\Re$,

$$
\begin{aligned}
\iint_{D} \frac{\overline{\partial k}}{\partial z} \frac{\partial \dot{g}}{\partial z} d x d y-\iint_{D} \frac{\partial \bar{k}}{\partial z} \frac{\partial \dot{g}}{\partial \bar{z}} d x d y \\
=\iint_{D^{*}} \frac{\overline{\partial k}}{\partial z} \frac{\partial \dot{g}}{\partial z} d x d y-\iint_{D^{*}} \frac{\partial \bar{k}}{\partial z} \frac{\partial \dot{g}}{\partial \bar{z}} d x d y=0 .
\end{aligned}
$$

Since $(\dot{)})$ is dense in $\$$ we conclude that (2.3) also holds.

From this identity we immediately get

THEOREM 2.7. The space $\mathbb{B}$ is orthogonal to $\mathfrak{F}$ and to $\overline{\mathfrak{F}}: \circlearrowleft \perp \mathfrak{F}, \circlearrowleft \perp \overline{\mathfrak{F}}$.

Further we prove the decisive

TheOREM 2.8. The spaces $\mathfrak{F}, \overline{\mathfrak{F}}$, (S) span the whole space $\mathfrak{\Re}: \mathfrak{F} \oplus \overline{\mathfrak{F}} \oplus \mathfrak{S}=\mathfrak{F}$.

We construct $\dagger$ the following function $\dot{g}$ in $\dot{s}$ :

Let $\left|z-z_{0}\right| \leqq R$ be a circle within $D, z^{\prime}$ a point within this circle and $\left|z-z^{\prime}\right| \leqq r$ a circle in the interior of $\left|z-z_{0}\right| \leqq R$. Then we put

$$
\dot{g}(x, y)= \begin{cases}0 & \text { for }\left|z-z_{0}\right| \geqq R ; \\ \frac{1}{\pi} \log \mid \frac{R\left(z-z^{\prime}\right)}{R^{2}-\overline{\left(z^{\prime}-z_{0}\right)}\left(z-z_{0}\right)} & \text { for }\left|z-z_{0}\right| \leqq R, \\ \frac{1}{\pi} \log \mid \frac{R r}{R^{2}-\overline{\left(z^{\prime}-z_{0}\right)}\left(z-z_{0}\right)} & \text { for }\left|z-z^{\prime}\right| \leqq r .\end{cases}
$$

Let $k$ be a function orthogonal to (s). Then from $(i g \mid k)=0$ and $(g \mid k)=0$ we

$\dagger$ For the following reasoning cf. G. Fubini, loc. cit., p. 10, $\S 6,7$, and R. Courant, Über direkte Methoden, bei Variations- und Randwertproblemen, Jahresbericht der Deutschen mathematiker vereinigung, vol. 34 (1925), pp. 107, 108. 
have $\iint_{D}(d k / d z)(d \bar{g} / d \bar{z}) d x d y=0$, according to (2.3). Hence we obtain by integration by parts

$$
\frac{1}{2 \pi i} \int_{\left|z-z^{\prime}\right|=r} k(x, y) \frac{d z}{z-z^{\prime}}=\frac{1}{2 \pi i} \int_{\left|z-z_{0}\right|=R} k(x, y) \frac{R^{2}-\left|z^{\prime}-z_{0}\right|^{2}}{\left|z-z^{\prime}\right|^{2}} \frac{d z}{z-z_{0}} .
$$

Consequently the mean value at the left-hand side is independent of $r$ and is a potential function $k\left(x^{\prime}, y^{\prime}\right)$ in $x^{\prime}, y^{\prime}$. The function $k^{*}(x, y)-k(x, y)$ has the property that

$$
\frac{1}{2 \pi i} \int_{\left|z-z^{\prime}\right|=r}\left(k^{*}-k\right) \frac{d z}{z-z^{\prime}}=0
$$

and, consequently,

$$
\iint_{\left|z-z^{\prime}\right| \leqq r}\left(k^{*}-k\right) d x d y=0 .
$$

Therefore $\nmid \iint_{D}\left|k^{*}-k\right|^{2} d x d y=0$ and $k$ is equivalent to the potential function $k^{*}$ (cf. Lemma 2.1). According to Theorem 2.5 the element $k$ belongs to $\mathfrak{F} \oplus \overline{\mathfrak{F}}$. Thus $\Re=\mathfrak{F} \oplus \overline{\mathfrak{F}} \oplus ß)$ as we wished to prove.

We denote by $F, \bar{F}, G$ the orthogonal projectors which belong to the closed subspaces $\mathfrak{F}, \overline{\mathfrak{F}}, \mathfrak{S}$; the projections of a function $k$ of $\Omega$ on these spaces are $F k, \bar{F} k, G k$ respectively. Theorem 2.8 gives the relation

$$
F+\bar{F}+G=1 \text {. }
$$

3. The SPACE $\mathfrak{S}$

3.1. The metric (,). In this section we deal with the space

$$
\mathfrak{S}=\mathfrak{F}+\mathfrak{B}
$$

consisting of all elements $h$ of $\Re$ which are orthogonal to $\overline{\mathfrak{F}}$. We employ the abbreviation

for all $k$ in $\Omega$, so that

$$
\begin{aligned}
& \left(k_{1}, k_{2}\right)=\Re \iint_{D} \frac{\overline{\partial k_{1}}}{\partial z} \frac{\partial k_{2}}{\partial z} d x d y \\
& \left(k_{1} ; k_{2}\right)=\Re \iint_{D} \frac{\partial \bar{k}_{1}}{\partial z} \frac{\partial k_{2}}{\partial \bar{z}} d x d y
\end{aligned}
$$

$\dagger$ We make use of the

LEMMA 2.2. Whenever an $L^{2}$-integrable function $\phi(x, y)$ has the property that

$$
\frac{1}{r^{2} \pi} \iint_{\left|z^{\prime}-z\right| \leqq r} \phi\left(x^{\prime}, y^{\prime}\right) d x^{\prime} d y^{\prime}=: 0
$$

for every circle $\left|z-z^{\prime}\right| \leqq r$ within $D$, then $\iint_{D}|\phi|^{2} d x d y=0$. 


$$
\left(k_{1}, k_{2}\right)+\left(k_{1} ; k_{2}\right)=\left(k_{1} \mid k_{2}\right) \text {. }
$$

The inner product $\left(h_{1}, h_{2}\right)$ defines a new metric $($,$) in the space \mathfrak{E}$, since the form $(h, h)$ is positive definite and vanishes for $h$ in $\mathfrak{W}$ only if $h=0$. An immediate consequence of identities (2.1) and (2.3) is

THEOREM 3.1. The spaces $\mathfrak{F}$ and $\mathbb{S}$ are orthogonal with respect to the metric (,).

Further we use

Lemma 3.1. For elements $h$ in $\$$ the inequality

is valid.

$$
\frac{1}{2}(h \mid h) \leqq(h, h) \leqq(h \mid h)
$$

From the identities (2.2) and (2.3), on writing $h=F h+G h=f+g$, we get in fact the relation

$$
\begin{aligned}
\frac{1}{2}(h \mid h) & =\frac{1}{2}(f \mid f)+\frac{1}{2}(g \mid g)=\frac{1}{2}(f, f)+(g, g) \\
\leqq & \leqq(f, f)+(g, g)=(h, h) \leqq(h, h)+(h ; h)=(h \mid h) .
\end{aligned}
$$

From this inequality we deduce

Lemma 3.2. A subspace of $\mathfrak{S}$ is complete with respect to the metric $(\mid)$ if and only if it is complete with respect to the metric (,).

Thus we obtain

THEOREM 3.2. The spaces $\mathfrak{F}$ and \& are closed with respect to (,).

In the following part of $\S 3$ the terms "orthogonal" and "closed" refer to the metric (,) only.

The projectors $F$ and $G$ for the spaces $\mathfrak{F}$ and $(5)$ satisfy the relation

$$
F+G=1 \text { in } \mathfrak{S} \text {. }
$$

They belong to the form $()-,(;)$ and $(;)$ in the sense that

$$
\left(h^{*}, F h\right)=\left(h^{*}, h\right)-\left(h^{*} ; h\right), \quad\left(h^{*}, G h\right)=\left(h^{*} ; h\right)
$$

for $h^{*}, h$ in $\mathfrak{S}$, in accordance with (2.3).

3.2. The spaces $\mathfrak{B}$ and $\mathfrak{Q}$. We introduce the symmetric forms

$$
h_{1} P h_{2}=\frac{1}{4} \iint_{D}\left(\frac{\overline{\partial h_{1}}}{\partial z}+\frac{\partial h_{1}}{\partial z}\right)\left(\frac{\partial h_{2}}{\partial z}+\frac{\overline{\partial h_{2}}}{\partial z}\right) d x d y=\iint_{D} \Re \frac{\partial h_{1}}{\partial z} \Re \frac{\partial h_{2}}{\partial z} d x d y
$$




$$
h_{1} Q h_{2}=\frac{1}{4} \iint_{D}\left(\frac{\bar{\partial} h_{1}}{\partial z}-\frac{\partial h_{1}}{\partial z}\right)\left(\frac{\partial h_{2}}{\partial z}-\frac{\overline{\partial h_{2}}}{\partial z}\right) d x d y=\iint_{D} \Im \frac{\partial h_{1}}{\partial z} \Im \frac{\partial h_{2}}{\partial z} d x d y
$$

defined for $h_{1}, h_{2}$ in $\mathfrak{S}$. We have

$$
0 \leqq h P h \leqq(h, h), \quad 0 \leqq h Q h \leqq(h, h)
$$

and

$$
\boldsymbol{P}+\boldsymbol{Q}=(,) \text {. }
$$

The forms $P$ and $Q$ correspond to bounded symmetric operators $P, Q$ such that

$$
h^{*} P h=\left(h^{*}, P h\right) ; \quad h^{*} Q h=\left(h^{*}, Q h\right)
$$

for $h^{*}, h$ in $\mathfrak{S} . P$ and $Q$ satisfy the relation

$$
P+Q=1 \text {. }
$$

By $\mathfrak{B}$ and $\mathfrak{Q}$ we denote the subspaces of all the elements $p$ and $q$ in $\mathfrak{S}$ for which

respectively.

$$
Q p=0 \text { and } P q=0
$$

The functions

$$
p=p_{x}+i p_{y} \text { in } \mathfrak{B} \text { and } q=q_{x}+i q_{\nu} \text { in } \mathfrak{Q}
$$

can be characterized as well by

$$
p Q p=\iint_{D}\left(\Im \frac{\partial p}{\partial z}\right)^{2} d x d y=\frac{1}{4} \iint_{D}\left(\frac{\partial p_{y}}{\partial x}-\frac{\partial p_{x}}{\partial y}\right)^{2} d x d y=0
$$

and

$$
q P q=\iint_{D}\left(\Re \frac{\partial q}{\partial z}\right)^{2} d x d y=\frac{1}{4} \iint_{D}\left(\frac{\partial q_{x}}{\partial x}+\frac{\partial q_{y}}{\partial y}\right)^{2} d x d y=0
$$

respectively. We have

TheOREM 3.3. The spaces $\mathfrak{B}$ and $\mathfrak{Q}$ are closed.

Since $p$ in $\mathfrak{B}, q$ in $\mathfrak{Q}$

$$
(p, q)=p P q+p Q q=(p, P q)+(Q p, q)=0
$$

we have

THEOREM 3.4. The spaces $\mathfrak{B}$ and $\mathfrak{Q}$ are orthogonal: $\mathfrak{B} \perp \mathfrak{Q}$.

We now prove the basic

Theorem 3.5. The spaces $\mathfrak{B}$ and $\mathfrak{Q}$ span the whole space $\mathfrak{S}: \mathfrak{P} \oplus \mathfrak{Q}=\mathfrak{W}$. 
Let $h$ be a function in $\mathfrak{S}$ which is orthogonal to $\mathfrak{B}$. Then we have

$$
p P h=p P h+p Q h=(p, h)=0 .
$$

We take the function

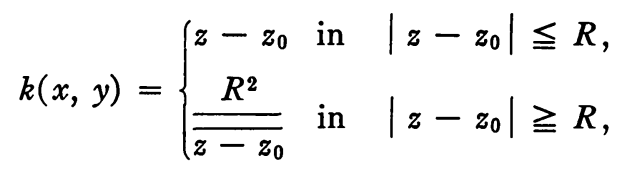

which belongs to $\Omega$ and has the property

$$
\frac{\partial k}{\partial z}=1 \quad \text { in }\left|z-z_{0}\right|<R, \quad=0 \text { in }\left|z-z_{0}\right|>R .
$$

The projection of this function on the space $\mathfrak{E}: p=k-\bar{F} k$ also has the property

$$
\frac{\partial p}{\partial z}=1 \quad \text { in }\left|z-z_{0}\right|<R, \quad=0 \text { in }\left|z-z_{0}\right|>R
$$

and, therefore, belongs to $\mathfrak{B}$. By inserting this function $p$ into the relation (3.1) we get

$$
\Re \iint_{\left|z-z_{0}\right|<R} \frac{\partial h}{\partial z} d x d y=0 .
$$

Since the circle $\left|z-z_{0}\right|<R$ was arbitrary within $D$, we deduce (cf. Lemma 2.2)

$$
\iint_{D}\left(\Re \frac{\partial h}{\partial z}\right)^{2} d x d y=0 .
$$

Hence $h$ belongs to $\mathfrak{Q}$ and consequently $\mathfrak{B} \oplus \mathfrak{Q}=\mathfrak{W}$.

From Theorem 3.5 we see that the elements $Q h$ belong to $\mathfrak{Q}$ because they are orthogonal to $\mathfrak{B}:(p, Q h)=(Q p, h)=0$. Thus we have $P Q=0$ or $Q^{2}=Q$; in the same way we find $P^{2}=P$. Therefore we have

TheOREM 3.6. The operators $P$ and $Q$ are the projectors of the spaces $\mathfrak{B}$ and $\mathfrak{Q}$ respectively.

Hence we see that the theory of $\$ 1$ is applicable.

3.3. The section spaces. Before going into detail we investigate the section spaces $\mathfrak{B F}, \mathfrak{Q F}, \mathfrak{B S}, \mathfrak{Q} S$.

We have

$$
\mathfrak{B F}=\{z\}, \quad \mathfrak{Q F}=\{i z\}
$$


for $f=z+$ const. and $f=i z+$ const. are the only functions of $\mathfrak{F}$ for which $d f / d z$ is real and imaginary respectively. The spaces $\mathfrak{B S}$ and $\mathfrak{Q} \$$ consist of all functions of $\mathfrak{B}$ and $\mathfrak{Q}$, respectively, which are constant at the boundary in the approximate sense of the definition of $\mathbb{S}$ (cf. $\$ 2$ ). Let $\mathfrak{F}^{\prime}, \mathfrak{S}^{\prime}, \mathfrak{B}^{\prime}, \mathfrak{Q}^{\prime}$ be the subspaces of all functions in $\mathfrak{F}, \mathfrak{S}, \mathfrak{B}, \mathfrak{Q}$ which are orthogonal to the section spaces; then we have $\mathfrak{S}^{\prime}=\mathfrak{F}^{\prime} \oplus \mathfrak{S}^{\prime}=\mathfrak{B}^{\prime} \oplus \mathfrak{Q}^{\prime}$. We establish the following theorem, but we shall not make use of it.

THEOREM 3.7. The elements $h$ of $\mathfrak{S}^{\prime}$ are equivalent to functions of the form

$$
h=z \overline{\phi_{1}(z)}+\phi_{2}(z)+\overline{\phi_{0}(z)},
$$

where $\phi_{1}(z), \phi_{2}(z)$, and $\phi_{0}(z)$ are analytic in $z$.

To prove this we take a circle $\left|z-z_{0}\right| \leqq 2 R$ in $D$; we choose a real function $\phi(x, y)$ which is four times continuously differentiable and which is $=1$ in $\left|z-z_{0}\right| \leqq R,=0$ in $\left|z-z_{0}\right| \geqq 2 R$. Then we choose a number $r<R$ and a point $z^{\prime}$ of $\left|z^{\prime}-z_{0}\right|<R-r$ and set

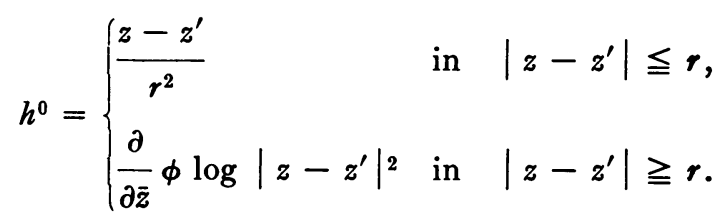

This function $h^{0}$ belongs to $\mathfrak{B S}$ and $i h^{0}$ belongs to $\mathfrak{Q} S$. Therefore $\left(h^{0}, h\right)$ $=\left(i h^{0}, h\right)=0$. This implies

$$
\frac{1}{r^{2} \pi} \iint_{\left|z-z^{\prime}\right| \leqq r} \frac{\partial h}{\partial z} d x d y=-\frac{1}{\pi} \iint_{R \leqq\left|z-z_{0}\right| \leqq 2 R} \frac{\partial^{2}}{\partial z \partial \bar{z}} \phi \log \left|z-z^{\prime}\right|^{2} \frac{\partial h}{\partial z} d x d y .
$$

The integral on the right is a potential function in $x^{\prime}, y_{1}^{\prime}$ which is independent of $r$ and can be written in the form $\overline{\phi_{1}\left(z^{\prime}\right)}+\phi_{2}^{\prime}\left(z^{\prime}\right)$, where $\phi_{1}(\bar{z})$ and $\phi_{2}(z)$ are analytic in $z$. On setting

$$
\phi^{*}=z \overline{\phi_{1}(z)}+\phi_{2}(z)
$$

we get

$$
\iint_{\left|z-z^{\prime}\right| \leqq r} \frac{\partial}{\partial z}\left(h-\phi^{*}\right) d x d y=0
$$

and (cf. Lemma 2.2)

$$
\iint_{D}\left|\frac{\partial\left(h-\phi^{*}\right)}{\partial z}\right|^{2} d x d y=0 .
$$


Therefore $\overline{h-\phi^{*}}$ is equivalent to an analytic function $\phi_{0}(z)$, and $h$ itself is equivalent to $z \overline{\phi_{1}(z)}+\phi_{2}(z)+\overline{\phi_{0}(z)}$.

3.4. The operator $M$. In $\$ 1$ we investigated the operator $F Q F$ in $\mathfrak{F}$. In its place, we now consider the bounded symmetric operator

$$
M=F(P-Q) F=F-2 F Q F,
$$

which takes every element $f$ of $\mathfrak{F}$ into the element $M f$ in $\mathfrak{F}$. This operator $M$ in $\mathfrak{F}$ belongs to the form

$$
f_{1} M f_{2}=f_{1} P f_{2}-f_{1} Q f_{2}=\Re \iint_{D} \frac{d f_{1}}{d z} \frac{d f_{2}}{d z} d x d y
$$

in the sense that

$$
f_{1} M f_{2}=\left(f_{1}, M f_{2}\right) .
$$

Now, this form $M$ is exactly the form dealt with in Part I if $w=d f / d z$; and thus we obtain a very simple representation of the operator of this form. On the other hand we can use the properties of this form established in Part I to investigate the new forms $P$ and $\boldsymbol{Q}$.

\section{THE FORM $E$}

4.1. The inequality. In this section we assume, as in Part $I$, that the boundary $B$ of the domain $D$ consists of a finite number of curves $z=z(s)$ with continuous tangent $z(s)$ except at a finite number of corners where $\dot{z}(s)$ is continuous on each side and $\operatorname{arc} \dot{z}(s)$ jumps by less than $\pi$. Then we get (in the sense of $\$ 1$ )

TheOREM 4.1. The spaces $\mathfrak{F}$ and $\mathfrak{Q}$ (or $\mathfrak{B}$ ) have a positive smallest angle.

Under our assumption on the boundary we can deduce from Theorem 1, Part I that there is a constant $\theta<1$ such that for all functions $f$ in $\mathfrak{F}$ satisfying the relation

the inequality

$$
\Re \iint_{D} \frac{d f}{d z} d x d y=0
$$

$$
f \boldsymbol{M} f=\Re \iint_{D}\left(\frac{d f}{d z}\right)^{2} d x d y \leqq \theta \iint_{D}\left|\frac{d f}{d z}\right|^{2} d x d y
$$

is valid. Since the form $M$ at the left-hand side belongs to the operator $M=1-2 F Q F$ in $\mathfrak{F}$ we get, on setting if instead of $f$, the inequality

$$
-(f, f)+2(f, Q f) \leqq \theta(f, f)
$$

or 


$$
(f, Q f) \leqq \frac{1+\theta}{2}(f, f),
$$

for all functions $f$ in $\mathfrak{F}$ which satisfy the relation

$$
(i z, f)=0
$$

and which are therefore orthogonal to $\mathfrak{Q F}=\{i z\}$. (In the same way we get $(f, P f) \leqq(1+\theta) / 2(f, f)$ under $(z, f)=0$. $)$ Therefore $\cos ^{2} \tau_{0} \leqq(1+\theta) / 2<1$ and the smallest angle $\tau_{0}<\pi / 2$ is positive as we stated in Theorem 4.1.

We introduce the form

$$
\begin{aligned}
h_{1} E h_{2} & =a\left(h_{1}, P h_{2}\right)+b\left(h_{1}, G h_{2}\right)=a\left(h_{1} P h_{2}\right)+b\left(h_{1} ; h_{2}\right) \\
& =a \iint_{D} \Re \frac{\partial h_{1}}{\partial z} \Re \frac{\partial h_{2}}{\partial z} d x d y+b \Re \iint_{D} \frac{\partial \bar{h}_{1}}{\partial z} \frac{\partial h_{2}}{\partial \bar{z}} d x d y
\end{aligned}
$$

for $h$ in $\mathfrak{S}$. We assume $a$ and $b$ to be positive. Applying Theorem 1.1 of $\$ 1$ we deduce immediately from Theorem 4.1 the

THEOREM 4.2. There is a positive constant $\rho$ such that

$$
\rho(h, h) \leqq(h E h)
$$

or

$$
\rho \iint_{D}\left|\frac{\partial h}{\partial z}\right|^{2} d x d y \leqq a \iint_{D}\left|\Re \frac{\partial h}{\partial z}\right|^{2} d x d y+b \iint_{D}\left|\frac{\partial h}{\partial \bar{z}}\right|^{2} d x d y
$$

for all functions $h$ in $\Re$ which satisfy the condition

$$
(i z, h)=\Im \iint_{D} \frac{\partial h}{\partial z} d x d y=0 .
$$

We now consider the form

$$
k_{1} E k_{2}=a \iint_{D} \Re \frac{\partial k_{1}}{\partial z} \Re \frac{\partial k_{2}}{\partial z} d x d y+b \Re \iint_{D} \frac{\partial \bar{k}_{1}}{\partial z} \frac{\partial k_{2}}{\partial \bar{z}} d x d y
$$

for $k$ in $\Re ; a>0, b>0$. On setting $k=(F+G) k+\bar{F} k=h+\bar{f}$, we get

(cf. Lemma 3.1), and

$$
\begin{aligned}
k_{1} E k_{2} & =h_{1} E h_{2}+b\left(\bar{f}_{1} \mid \bar{f}_{2}\right) \\
\left(k_{1} \mid k_{2}\right) & =\left(h_{1} \mid h_{2}\right)+\left(\bar{f}_{1} \mid \bar{f}_{2}\right) \\
& \leqq 2\left(h_{1}, h_{2}\right)+\left(\bar{f}_{1} \mid \bar{f}_{2}\right)
\end{aligned}
$$

$$
(i z \mid k)=(i z, h) .
$$

Therefore we obtain from Theorem 4.2 
THEOREM 4.3. There is a positive constant $\sigma$ such that

$$
\sigma(k \mid k) \leqq(k E k),
$$

or

$\sigma \iint_{D}\left|\frac{\partial k}{\partial z}\right|^{2} d x d y+\sigma \iint_{D}\left|\frac{\partial k}{\partial \bar{z}}\right|^{2} d x d y \leqq a \iint_{D}\left(\Re \frac{\partial k}{\partial z}\right)^{2}+b \iint_{D}\left|\frac{\partial k}{\partial \bar{z}}\right|^{2} d x d y$,

for all functions $k$ in $\Re$ which satisfy the condition

$$
(i z \mid k)=\Im \iint_{D} \frac{\partial k}{\partial z} d x d y=0 .
$$

4.2. The spectral resolution. It is very simple to give the spectral resolution of the form $E$ with respect to the unit-form (,) or $(\mid)$ if we assume that the boundary $B$ has no corners.

First we remark that, according to Theorem 4 of Part I, the operator $M=1-2 F Q F$ in $\mathfrak{F}$ has a pure point spectrum of values

$$
\mu_{1}=1>\mu_{2} \geqq \mu_{3} \geqq \cdots \rightarrow 0 ; \quad \mu_{-n}=-\mu_{n} .
$$

Therefore the operator $F Q F$ in $\mathfrak{F}$ also has a pure point spectrum of values

$$
\kappa_{n}=\frac{1-\mu_{n}}{2} \rightarrow \frac{1}{2}, \quad n= \pm 1, \pm 2, \pm 3, \cdots .
$$

The characteristic functions of $F Q F$ and $M$ are the same $f_{n}(z) ; f_{-n}(z)=i f_{n}(z)$. $f_{1}(z)=a z, f_{-1}(z)=i a z ; a=$ real constant.

We now observe that the form $E$ in $\mathfrak{S}$ belongs to the operator $a P+b G$ with respect to the unit-form (,). Therefore we can apply Theorem 1.3 of $\S 1$. We find that this operator has a pure point spectrum of values $\lambda_{n}^{+}, \lambda_{n}^{-}$, $n= \pm 1, \pm 2, \cdots$, which are the solutions of the quadratic equation

$$
\left(\lambda_{n}-a\right)\left(\lambda_{n}-b\right)=a b \frac{1-\mu_{n}}{2} .
$$

For $n= \pm 1$ we get the characteristic values $\lambda=0, a, b, a+b$; their characteristic spaces are

$$
\mathfrak{Q F}=\{i z\}, \quad \mathfrak{B F}=\{z\}, \quad \mathfrak{Q B S}, \quad \mathfrak{B S} .
$$

The characteristic functions of $\lambda_{n}$ for $n= \pm 2, \pm 3, \cdots$ are

$$
h_{n}=\left(\lambda_{n}-b\right) f_{n}-a Q f_{n} .
$$

They belong to the space $\mathfrak{S}^{\prime}$ and span it. 
The set of the characteristic values $\lambda_{n}^{+}, \lambda_{n}^{-}$has the two limit points

$$
\lambda_{\infty}^{+}=\frac{a+b}{2}+\frac{1}{2}\left[a^{2}+b^{2}\right]^{1 / 2}, \quad \lambda_{\infty}^{-}=\frac{a+b}{2}-\frac{1}{2}\left[a^{2}+b^{2}\right]^{1 / 2} .
$$

To give the spectral resolution of the form $k_{1} E k_{2}$ with respect to the unitform $(\mid)$ we observe the relations

$$
\begin{aligned}
k_{1} E k_{2} & =h_{1} E h_{2}+b\left(\bar{f}_{1} \mid \bar{f}_{2}\right), \\
\left(k_{1} \mid k_{2}\right) & =\left(h_{1}, F h_{2}\right)+2\left(h_{1}, G h_{2}\right)+\left(\bar{f}_{1} \mid \bar{f}_{2}\right) .
\end{aligned}
$$

Therefore the space $\overline{\mathfrak{F}}$ is characteristic with value $b$. The other characteristic functions belong to $\mathfrak{E}$; to find them we apply the remarks at the end of $\$ 1$ and get the following:

The values $\lambda=0, a, b / 2,(a+b) / 2, b$ are characteristic with spaces $\mathfrak{O F}=\{i z\}, \mathfrak{B F}=\{z\}, \mathfrak{O G}, \mathfrak{B S}, \mathfrak{F}$. The other characteristic values $\lambda_{n}^{+}, \lambda_{n}^{-}$, $n= \pm 2, \pm 3, \cdots$, are the solutions of the equation

$$
\left(\lambda_{n}-a\right)\left(2 \lambda_{n}-b\right)+\left(\lambda_{n}-b\right) a \frac{1-\mu_{n}}{2} ;
$$

they have two limit points $\lambda_{\infty}^{+}, \lambda_{\infty}^{-}$, solutions of

$$
\left(\lambda_{\infty}-a\right)\left(2 \lambda_{\infty}-b\right)+\left(\lambda_{\infty}-b\right) \frac{a}{2}=0 .
$$

Their characteristic functions belong to the space $\mathfrak{S}^{\prime}$ and span it.

\section{Application to the theory of the elastic plate}

Finally we outline the application of the inequality of Theorem 4.2 to the theory of an elastic plate. Let us imagine an elastic plate spread out over the domain $D$. We assume that the boundary $B$ of $D$ consists of a finite number of curves with a continuous tangent except at a finite number of corners as in $\S 4$. Let $k(x, y)$ be the displacement transforming every point $z$ of $D$ into the point $z+k$. Then the potential energy arising from this deformation $\dagger$ is

$\dagger$ The theory of transversal displacements also depends on the form $k E k$ provided that $k$ is the gradient $k=\partial j / \partial z$ of a real function $j(x, y)$. The equilibrium problem has been treated several times with the help of variational methods. For the first time by G. Fubini, loc. cit., and by W. Ritz, Journal für die reine und angewandte Mathematik, vol. 135 (1909). For the vibrations see W. Ritz, Annalen der Physik (1909). For both see K. Friedrichs, Mathematische Annalen, vol. 98 (1927), pp. 206-247. For these problems, where $k=\partial j / \partial z$ belongs to $\mathfrak{B}+\bar{F}$, our inequality (Theorem 4.2) need not be used if $b \neq 0$. 


$$
k E k=a \iint_{D}\left(\Re \frac{\partial k}{\partial z}\right)^{2} d x d y+b \iint_{D}\left|\frac{\partial k}{\partial z}\right|^{2} d x d y,
$$

where $a$ and $b$ are positive constants. $\dagger$

We restrict $k$ to the subspace $\Omega_{0}$ of one-valued functions in $\Re$. In $\Omega_{0}$ we introduce the form

$$
k_{1} H k_{2}=\Re \iint_{D} \bar{k}_{1} k_{2} d x d y .
$$

Then the kinetic energy arising from the vibration $k e^{i \omega t}$ is proportional to

$$
k H k=\iint_{D}|k|^{2} d x d y .
$$

Let the complex-valued function $\phi(x, y)$ be the density of a force applied over the interior of $D$; the potential energy of this force is

$$
-k H \phi=-\Re \iint_{D} \bar{k} \phi d x d y .
$$

There are different cases according as the displacement $k$ has to satisfy boundary conditions or not.

First we take the case (1) of no displacement at the boundary $B$. Then the displacement $g$ belongs to the subspace (\$).

The problem of equilibrium (1) is: to find a displacement $\underline{g}$ in $\&$ such that

$$
2 g^{\prime} E \underline{g}-g^{\prime} H \phi=0
$$

for all $g^{\prime}$ in (S).

The theory of vibrations (1) requires the simultaneous spectral resolution of the forms $\mathrm{g} \mathrm{Eg}$ and $\mathrm{gHg}$.

Now, for $g$ in (\$) we have the relation

$$
g E g \geqq b \iint_{D}\left|\frac{\partial g}{\partial \bar{z}}\right|^{2} d x d y=\frac{b}{2}(g \mid g) .
$$

Since the form $H$ in $\&$ is bounded and completely continuous with respect to $(\mid)$, it has like properties with respect to $E$. Therefore no difficulty arises in solving the two problems.

In case (2) there is no boundary condition for the displacement (and no force working at the boundary).

t It is

$$
a=\frac{m+1}{m-1} G=\frac{2 m}{m-1} E, \quad b=G=\frac{2 m}{m-1} E
$$

where $E, G, m$ are the moduls of elasticity. 
The question of equilibrium (2) is: to find a displacement $\underline{k}$ in $\Omega_{0}$ such that

$$
2 k^{\prime} E \underline{k}-k^{\prime} H \phi=0
$$

for all $k^{\prime}$ in $\Omega^{\prime}$. Here we must assume $1 H \phi=0, i H \phi=0$, that is, $\iint_{D} \phi d x=0$ and $i z H \phi=\Im \iint_{D} \bar{z} \phi d x d y=0$ as we shall see.

The theory of vibrations (2) requires the simultaneous spectral resolution of the forms $k E k$ and $k H k$ for $k$ in $\Omega_{0}$.

In the case (2) the energy $k E k$ vanishes for a pure translation $k=$ const. and a pure rotation $k=i z$. To exclude this we take the accessory conditions

$$
\begin{aligned}
& 1 H k=0, \quad i H k=0 \quad \text { or } \quad \iint_{D} k d x d y=0 \\
& i z H k=0 \quad \text { or } \quad \Im \iint_{D} \bar{z} k d x d y=0 .
\end{aligned}
$$

By $\Omega_{1}$ or $\Omega_{2}$ we denote the space of all functions $k$ in $\Omega_{0}$ satisfying (5.1) or (5.1) and (5.2) respectively. In $\Omega_{1}$ and $\Omega_{2}$ we use the unit-form (|).

We employ the inequality of Poincaré: $\dagger$

There is a positive constant $\pi$ such that for all $k$ in $\Re_{1}$

$$
k H k \leqq \pi(k \mid k) \text {. }
$$

So the form $H$ is bounded in $\Re_{1}$. Therefore the spaces $\Re_{1}$ and $\Omega_{2}$ are closed with respect to $(\mid)$.

Further we note $\dagger$

The form $H$ is completely continuous in $\Omega_{1}$, with respect to $(\mid)$.

Now we prove

THEOREM 5.1. There is a positive constant $\epsilon$ such that for all functions $k$ in $\Omega_{2}$

$$
\epsilon(k \mid k) \leqq(k E k) .
$$

Since the space $\{i z\}$ has the dimension 1 it has a positive smallest angle $\tau_{0} \leqq \pi / 2$ with respect to the space $\Omega_{2}$ and the section $\{i z\} \Omega_{2}=0$. Let aiz be the projection of $k$ into $\{i z\}$; then we have (cf. $(1.2)_{P}$ ) for $k$ in $\Omega_{2}$

$$
(k-a i z \mid k-a i z) \geqq \sin ^{2} \tau_{0}(k \mid k) .
$$

Now, $k-a i z$ being orthogonal to $\{i z\}$, Theorem 4.3 gives a constant $\sigma>0$ such that

† Cf. e.g. K. Friedrichs, Spektraltheorie halbbeschränkter Operatoren und Anwendung auf die spektral Zerlegung von Differentialoperatoren. II. Mathematische Annalen, vol. 109 (1934), pp. 705707. 


$$
\sigma(k-a i z \mid k-a i z) \leqq(k-a i z) E(k-a i z) .
$$

But we have

$$
(k-a i z) \boldsymbol{E}(k-a i z)=(k E k) .
$$

Thus Theorem 5.1 is proved.

In consequence of Theorem 5.1 we can take $E$ as unit-form in the space $\Omega_{2}$, and we are sure that every subspace of $\Omega_{2}$ which is complete with respect to $(\mid)$ is complete with respect to $E$; especially $\Omega_{2}$ itself is closed as to $(\mid)$; further that every form in $\Omega_{2}$ which is bounded or completely continuous with respect to (|) has a like property with respect to $E$.

Now, since $k H \phi$ is a bounded linear form, it is a well known fact that there is a function $\underline{k}$ in $\Re_{2}$ such that

$$
2 k^{\prime} E \underline{k}-k^{\prime} H \phi=0
$$

for all $k^{\prime}$ in $\Re_{2}$; but since we have assumed $(a+i b) H \phi=0, i z H \phi=0$, this relation holds for all $k$ in $\Re_{0}$. This function $\underline{k}$ is the solution of the equilibrium problem (2).

The form $H$ is completely continuous with respect to $E$. From this we get the solution of the vibration problem (2):

There is a sequence of values $\eta_{1} \leqq \eta_{2} \leqq \eta_{3} \leqq \cdots \rightarrow \infty$ and of functions $k_{1}, k_{2}, k_{3}, \cdots$ in $\Re_{2}$ orthogonal with respect to $\boldsymbol{H}$ and $E$ such that every function $k$ in $\Re_{2}$ can be developed into a series

$$
k=a_{1} k_{1}+a_{2} k_{2}+a_{3} k_{3}+\cdots
$$

by real coefficients $a_{1}, a_{2}, a_{3}, \cdots$ in the sense that

$$
\begin{aligned}
& k H k=a_{1}^{2}+a_{2}^{2}+a_{3}^{2}+\cdots \\
& k E k=\eta_{1} a_{1}^{2}+\eta_{2} a_{2}^{2}+\eta_{3} a_{3}^{2}+\cdots .
\end{aligned}
$$

It is beyond our purpose to discuss the nature of the function $\underline{k}$ and of these characteristic functions in detail.

Braunschweig, Germany 